\title{
Research Paper \\ The Mediating Role of Alexithymia in the Relationship of Coping Strategies and Personality Traits With Obsessive-Compulsive Disorder
}

\author{
Masoud Bagheri ${ }^{1}$ (1), Kazem Nematollah Zadeh Mahani², "Maryam Pour Amrollahi ${ }^{2}$ (1)
}

1. Department of Psychology, Faculty of Literature and Humanities, Shahid Bahonar University of Kerman, Iran.

2. Department of Psychology, Faculty of Literature and Humanities, Islamic Azad University of Kerman, Iran.

$\begin{gathered}\text { Use your device to scan } \\ \text { and read the article online }\end{gathered}$
of Coping Strategies and Personality Traits With Obsessive-Compulsive Disorder (Persian)]. Quarterly of "The Horizon of Medical
Sciences". 2021; 27(1):62-81. https://doi.org/10.32598/hms.27.1.3355.1
doisttps://doi.org/10.32598/hms.27.1.3355.1

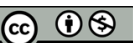

Received: 01 Mar 2020 Accepted: 20 Jul 2020 Available Online: 01 Jan 2021

Key words: Alexithymia, Coping, Personality, Obsession

\section{ABSTRACT}

Aims Obsessive-Compulsive Disorder (OCD) is one of the severe psychological health problems imposing considerable social and economic costs on society. OCD debilitating symptoms can disrupt interpersonal relations, job performance, and life quality. The purpose of this study is to investigate the mediating role of alexithymia in the relationship between coping strategies and personality traits with OCD.

Methods \& Materials This is a descriptive correlational study. The study population consists of all people with OCD referred to psychiatric clinics in Kerman City, Iran, during 2018-2019. Of this population, 200 patients were selected using a convenience sampling method. Research instruments included the ways of coping questionnaire, NEO five-factor personality inventory, Toronto alexithymia scale, and Maudsley obsessive-compulsive test. The obtained data were analyzed using the Pearson correlation test and simultaneous and hierarchical regression in SPSS V. 23.

Findings Personality traits had a negative significant correlation with alexithymia $(r=0.523, P<0.000)$ and $\operatorname{OCD}(r=0.253, P<0.000)$. Alexithymia had a significant positive correlation with $O C D(r=0.272, P<0.000)$. There was no correlation between problem-focused coping and alexithymia $(r=-0.045, P<0.531)$. There was a positive correlation between emotion-focused coping and OCD $(r=0.198, P<0.000)$. The fit indices indicated a good fit of the proposed model $(P<0.005)$.

Conclusion Alexithymia, as a mediator of the relationship between coping styles and personality traits with $O C D$, plays an essential role in improving the psychological health of people with OCD.

\section{Extended Abstract}

\section{Introduction}

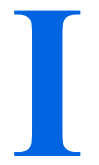

n today's complex world, human beings face many problems such as poverty, homelessness, unemployment, social unrest, war, rapid social changes, pressures of modern life, loneliness, personal deprivation, lack of support, incurable diseases, etc. Among all these problems, psychological problems are among the significant challenges of humans in the modern age. Among these psychological problems, ObsessiveCompulsive Disorder (OCD) creates discomfort and anxiety for the patients and their families because of causing many issues at the workplace, family, society, and the like [1]. This disorder is one of the most common psychiatric disorders and, with an estimated lifetime prevalence of $2 \%-3 \%$, is almost twice as common as schizophrenia and bipolar disorder. OCD is a chronic and debilitating disease that has two aspects: cognitive (intellectual) and behavioral (practical) [2]. Obsessive thoughts, impulses, and images

\section{* Corresponding Author:}

Maryam Pour Amrollahi

Address: Department of Psychology, Faculty of Literature and Humanities, Islamic Azad University of Kerman, Iran.

Tel: +98 (913) 3960137

E-mail: mashizi88@yahoo.com 
are disturbing and repetitive [3]. This disorder, with its appearance during childhood and early adulthood, has an early course of development, and if left untreated, it will get worse [4]. According to the Diagnostic and Statistical Manual of Psychiatric Disorders, the main characteristics of OCD are repetitive, unwanted thoughts and behaviors for a long time, causing significant distress. During this period, the patients recognize that their obsessions are unreasonable and excessive [6].

One of the variables related to OCD is personality traits $[1,7]$. Personality has different meanings [8]. Personality traits are a single organized set of relatively stable characteristics that distinguish one person from another [9]. Personality traits play an important role in effective emotional and physical functioning $[10,11]$. The traits that constitute the five-factor model are extraversion, neuroticism, openness to experience, agreeableness, and conscientiousness. Each of these five factors can be evolved out of the thoughts, feelings, and reactions of individuals separately [12]. According to Costa and McCrae [13], neuroticism refers to a person's tendency to experience anxiety, stress, compassion, hostility, impulsivity, depression, and low self-esteem. Extraversion refers to a person's tendency to be positive, assertive, energetic, and intimate. Openness to experience refers to a person's desire for curiosity, love of art, artistry, flexibility, and wisdom. Agreeableness refers to a person's willingness to generosity, kindness, empathy, sympathy, altruism, and trust. Finally, conscientiousness refers to a person's desire for orderliness, self-efficacy, dependability, self-discipline, progressiveness, reasonability, and calmness [14]. In a study of patients with OCD and depression, Rector et al. [15] concluded a modest relationship between neuroticism, extroversion, agreeableness, and openness to experience in both groups. Extraversion also had a positive relationship with agreeableness in both groups. While extraversion was not associated with conscientiousness in patients with OCD, there was a positive relationship between these dimensions in the depressed group. Finally, agreeableness in both groups was positively related to conscientiousness. Naghdi et al. [1] found a difference between the two study groups in the total score of neuroticism and its subscales of anxiety, depression, shyness, and vulnerability, where those with OCD got higher scores in this trait and subscales.

It seems that coping strategies are related to OCD. Coping strategies are a set of cognitive and behavioral efforts used to interpret and correct a stressful situation and reduce its suffering [16]. They are efforts that people make to overcome stressful situations according to their knowledge of the environment and behavior [17]. The more people can cope, the less likely they fall into stressful situations [18].
In other words, a person's vulnerability to stress is affected by coping skills and existing social support [19]. Problemfocused and emotion-focused coping strategies have been identified as two effective methods of dealing with stress [20]. In the problem-focused type, strategies are based on environmental conditions. They are used to remove or change situations that are considered a threat. Still, in the emotion-focused type, strategies are used to control the unpleasant feelings caused by stressful situations [21]. Studies have shown that coping skills are related to mental health [22, 23]. Coping styles reduce anxiety and stress, in contrast to ineffective coping styles that cause a loss of confidence in coping abilities and, as a result, develop anxiety, phobia, drug dependence, depression, and so on [24]. Sadock and Sadock [25] showed that coping strategies as negative thinking processes are among the underlying factors in the onset and persistence of OCD [25]. Sanderson also showed that an emotion-based coping strategy is associated with negative adjustment. Avoiding thinking about a traumatic event even prevents people from understanding the experience and eventually coping with it. Since the constant effort to avoid thinking about disturbing issues leads to chronic physiological arousal, reliance on this strategy reduces cellular immune activity [26]. Lazarus [27] believes that coping strategies have five tasks: Realistically coping with the problem, acting to adapt to adverse events, maintaining a positive self-image, maintaining emotional balance, and satisfying relationships with others [28]. Besharat et al.'s study to explain mental health based on problem-focused and emotion-focused coping styles in patients with multiple sclerosis revealed that a positive emotion-focused coping style has a positive relationship with psychological wellbeing and a negative association with psychological distress. Moreover, a negative emotion-focused coping style had a negative relationship with psychological wellbeing and a positive relationship with psychological distress. Wingenfeld et al. [30] found that emotion-focused coping styles against negative life events were positively associated with psychopathology, while problem-focused coping strategies were negatively correlated with psychopathology.

Another factor that plays a significant role in OCD is alexithymia [31]. Alexithymia is the difficulty in emotional self-regulation. In other words, it is the inability for cognitive processing of emotional information and regulating emotions [32]. This psychological variable is characterized by difficulty in identifying and describing feelings and externally-oriented thinking. These characteristics indicate deficiencies in cognitive processing and emotion regulation [33], especially when a task is not straightforward [34]. People with alexithymia exaggerate normal physiological arousal and misinterpret the somatic signs 
Table 1. Descriptive statistics of study variables

\begin{tabular}{|c|c|c|c|c|c|c|}
\hline Variables & Component & Mean \pm SD & Min. & Max. & Skewness & Kurtosis \\
\hline \multirow{5}{*}{ Problem-focused \pm coping } & Seeking \pm social \pm support & $9.755 \pm 3.13$ & 2 & 17 & 0.189 & -0.168 \\
\hline & Responsibility & $7.270 \pm 2.051$ & 3 & 12 & 0.308 & 0.265 \\
\hline & Planful \pm problem \pm solving & $8.385 \pm 2.730$ & 2 & 15 & -0.169 & -0.314 \\
\hline & Positive \pm reappraisal & $12.045 \pm 3.716$ & 2 & 20 & 0.177 & -0.204 \\
\hline & Total & $37.755 \pm 5.624$ & 24 & 54 & 0.616 & 0.755 \\
\hline \multirow{5}{*}{ Emotion-focused \pm coping } & Confrontive \pm coping & $8.370 \pm 2.738$ & 2 & 14 & -0.220 & -0.336 \\
\hline & Distancing & $9.135 \pm 2.483$ & 4 & 17 & 0.677 & 1.219 \\
\hline & Self-control & $11.440 \pm 2.707$ & 6 & 19 & 0.322 & 0.053 \\
\hline & Escape-avoidance & $10.96 \pm 3.756$ & 4 & 22 & 0.531 & 0.105 \\
\hline & Total \pm & $39.905 \pm 8.261$ & 18 & 64 & 0.281 & 1.012 \\
\hline \multirow{5}{*}{ Personality \pm traits } & Neuroticism & $21.725 \pm 7.325$ & 7 & 37 & 0.087 & -0.738 \\
\hline & Extraversion & $20.095 \pm 6.166$ & 6 & 34 & 0.187 & -0.182 \\
\hline & Openness \pm to \pm experience & $24.794 \pm 4.889$ & 14 & 34 & -0.156 & -0.401 \\
\hline & Agreeableness & $19.810 \pm 4.581$ & 10 & 30 & -0.077 & -0.238 \\
\hline & Conscientiousness & $16.750 \pm 4.637$ & 4 & 28 & -0.330 & 0.818 \\
\hline \multirow{4}{*}{ Alexithymia } & Difficulty \pm in \pm identifying \pm feelings & $18.930 \pm 5.503$ & 7 & 35 & 0.483 & 0.015 \\
\hline & Difficulty \pm in \pm describing \pm feelings & $13.565 \pm 3.961$ & 5 & 22 & -0.293 & -0.424 \\
\hline & Externally-orientedtthinking & $21.195 \pm 4.095$ & 10 & 33 & -0.253 & 0.579 \\
\hline & Total \pm & $53.690 \pm 9.514$ & 29 & 79 & -0.208 & 0.358 \\
\hline \multirow{5}{*}{ Obsessive \pm compulsive \pm disorder } & Checking & $3.985 \pm 2.247$ & 0 & 9 & 0.183 & -0.919 \\
\hline & Cleaning & $4.130 \pm 2.106$ & 0 & 9 & 0.313 & -0.568 \\
\hline & Slowness & $2.555 \pm 1.468$ & 0 & 6 & 0.314 & -0.216 \\
\hline & Doubting & $4.030 \pm 1.809$ & 1 & 12 & 0.453 & 0.625 \\
\hline & Total \pm & $13.170 \pm 3.350$ & 3 & 27 & 0.222 & -0.656 \\
\hline
\end{tabular}

of emotional arousal. Alexithymia is believed to be a risk factor for many psychiatric disorders because people with this condition are under a lot of pressure from physical/ emotional problems. This inability to regulate emotions makes successful coping difficult. People with emotional alexithymia engage in destructive nonverbal actions to express their feelings. Many scholars believe that psychological, social, and biological factors are involved in alexithymia [35]. Studies have shown that alexithymia has negative effects on psychological and emotional wellbeing and is a risk factor for people suffering from emotional distress, psychological problems, and mental illness [36, 37]. Besharat [38] and Grabe et al. [39] concluded that the OCD patients had higher alexithymia levels than the healthy group.

The inconsistent findings in studies that examined the relationship between $\mathrm{OCD}$ and other variables require further 
addressing this issue. The relationship of OCD with other anxiety symptoms, alexithymia, coping strategies, and personality disorders has been the subject of many studies with valuable findings. However, there are still many questions that need more studies and consideration of other factors. Considering this limitation and presenting the new components, the present study aimed to investigate the mediating role of alexithymia in the relationship between coping strategies and personality traits with OCD.

\section{Materials and Methods}

This research is a descriptive study using Structural Equation Modeling (SEM). The study population consists of all patients with diagnosed OCD referred to psychiatric clinics in Kerman City, Iran, in 2018-19. There is no information about the proportion of the population with OCD in Kerman, besides the sample size in SEM is influenced by several factors such as the distribution pattern of study variables in a multidimensional space (multivariate normality), the parameter estimation method (e.g. $\chi^{2}, \mathrm{CFI}, \mathrm{AGFI}$ ), the complexity of the model. Considering all these factors and the report of Hooman in 2008 and Klein in 2010 of a minimum sample size of 200 for structural models [40], the sample size of this study was determined as 200 . A convenience sampling method was used for selecting samples from the outpatients who had been referred for medication treatment for the first time or were in the early stages of treatment. The inclusion criteria were diagnosis of OCD based on clinical interview and confirmation of a psychiatrist, having at least 15 years of age, and at least a high school diploma. The exclusion criteria were having psychotic disorders, Tourette's disorder, drug dependence, epilepsy, organic psychosis, uncontrollable bipolar disorder; or using any type of psychiatric medication or psychological services.

\section{Data collection tools}

\section{The Ways of Coping Questionnaire}

The Ways of Coping Questionnaire (WCQ) is a 66-item self-report scale developed by Folkman and Lazarus [41] measuring 8 coping strategies in two categories of problemfocused (seeking social support, responsibility, planful problem solving, positive reappraisal) and emotion-focused strategies (confrontive coping, distancing, escape-avoidance, self-control). The items are rated on a 4-point Likert-type scale: $0=$ not used, $1=$ used somewhat, $2=$ used quite a bit, $3=$ used a great deal [41]. Folkman and Lazarus [41] reported internal consistency of 0.75 for WCQ and its subscales reliability from 0.61 for distancing to 0.79 for positive reappraisal. Aghayousefi [42] reported internal consistency of 0.80 for its Persian version. In our study, it was reported as 0.77 .

\section{NEO Fve-factor Inventory (NEO-FFI)}

NEO personality inventory was introduced by McCrae and Costa [13] with 185 items. Based on this original inventory, Its 250 -item and 60 -item forms were designed by them. In this study, we used the 60-item NEO-FFI with five dimensions of extraversion, neuroticism, openness to experience, agreeableness, and conscientiousness [13]. Its Persian version was first validated by Kiamehr [43] in a study on college students in Tehran. Each of five factors is measured by 12 items rated on a 5-point Likert scale from 0 to 4 (strongly disagree, disagree, no idea, agree, strongly agree). The final score of each subscale ranges from 0 to 48. McCrae and Costa [15] reported the Cronbach alpha coefficients of the 5 factors of neuroticism, extraversion, agreeableness, conscientiousness, and openness to experience as $0.84,0.82,0.78,0.65$, and 0.86 , respectively. For its Persian version, Abdollahzadeh reported the Cronbach alpha coefficients of the 5 factors as $0.67,0.59,0.64,0.76$, and 0.19 , respectively. In our study, they were obtained as $0.73,0.69,0.80,0.78$ and 0.81 , respectively.

\section{Toronto Alexithymia Scale}

Toronto Alexithymia Scale (TAS) was developed by Bagby et al. [44] and has 20 items and 3 subscales: difficulty in identifying feelings ( 7 items), difficulty in describing feelings (5 items), and externally-oriented thinking (8 items). The scoring is on a 5-point Likert scale ranged from 1 (strongly disagree) to 5 (strongly agree). The total score ranges from 20 to 100 . A score of 20-40 indicates a low level of alexithymia, a score of 40-60 indicates moderate alexithymia, and a score above 60 indicates a high level of alexithymia [44]. In the study by Besharat [45], the concurrent validity of the Persian version of TAS was examined and confirmed in terms of correlation with psychological wellbeing and psychological distress measurement tools. Moreover, the Cronbach alpha coefficients for total TAS score and its three subscales have been calculated 0.42-0.85, indicating a good internal consistency [38]. Its test-retest reliability for the total TAS score and its dimensions (0.70-0.77) with an interval of 4 weeks was confirmed [44]. In our study, its Cronbach alpha value was obtained at 0.71 .

\section{Maudsley Obsessive-Compulsive test}

Maudsley obsessive-compulsive test (MOCT) was developed by Rachman and Hodgson [46] with 30 statements answered by "Yes" or "No." In the initial validation phase at Maudsley Hospital, this test could distinguish 50 OCD patients from 50 neurotic patients. In the content analysis of the responses of 100 patients, 4 major components that 
reflected 4 types of OCD in patients were identified: checking, cleaning, slowness, and doubting [46]. This test has only two questions about obsessive thoughts and does not measure the extent and the severity of the problems facing them. However, it can distinguish patients with OCD from other neurotic people. Overall, MOCT is a useful and simple tool that can be used along with standard assessment methods. It is handy for assessing changes in treatment outcome, too [46]. It is a useful tool for examining the etiology, course, and prognosis of different obsessive complaint types. The test-retest reliability of this questionnaire has been reported as 0.89 [46].

After obtaining the patients' consent, questionnaires were given to them, and explanations were provided regarding their completion. Throughout the study, all ethical considerations related to the subjects were considered. They were free to participate in the research and were assured of the confidentiality of their information. During the study, no action or activity that can endanger the health of patients was taken. The obtained data were analyzed in SPSS V. 23 and AMOS V. 20 applications by using the SEM method.

\section{Results}

The participants were 112 males $(56 \%)$ and 88 females (44\%). Their age ranged from 18 to 45 years. A total of 38 $(19 \%)$ aged $18-25$ years; $38(19 \%)$ aged $26-30$ years; 45 (22.5\%) aged 35-31 years; $26(13 \%)$ aged $36-40$, and 53 $(26.5 \%)$ aged $41-45$ years. Moreover, 61 patients (30.5\%) had a high school diploma; 40 (20\%) a postgraduate degree; $58(29 \%)$ a Bachelor's degree, and 41 (20.5\%) a Master's degree. Descriptive statistics (mean, standard deviation, minimum, maximum, skewness, and kurtosis) of study variables are presented in Table 1. According to Klain in causal modeling, variables must be normal, and the absolute value of the skewness and kurtosis for the variables should not be more than 3 and 10, respectively. According to Table 1, the absolute value of skewness and kurtosis for all variables was less than these values; therefore, the assumption of normality was established.

The relationship between the variables was measured by the Pearson correlation test (Table 2). The results showed that the problem-focused coping strategy had a negative significant relationship with emotion-focused coping strategy (Sig. $=0.000 ; \mathrm{r}=-0.375$ ), neuroticism ( $\mathrm{Sig} .=0.003$; $\mathrm{r}=-0.206$ ), conscientiousness (Sig. $=0.001 ; \mathrm{r}=-0.233$ ), and alexithymia (Sig. $=0.000 ; \mathrm{r}=-0.275)$. But it had no significant relationship with OCD (Sig. $=0.531 ; \mathrm{r}=-0.045$ ), extraversion ( $\mathrm{Sig}=0.389$; $\mathrm{r}=-0.061$ ), openness to experience (Sig. $=0.375 ; \mathrm{r}=0.063$ ), and agreeableness (Sig. $=0.234$; $\mathrm{r}=-0.085$ ). Emotion-focused coping strategy had a significant negative relationship with neuroticism (Sig. $=0.000$; $\mathrm{r}=-0.453$ ), and a positive significant relationship with OCD (Sig. $=0.005 ; \mathrm{r}=0.198$ ), openness to experience (Sig. $=0.001 ; \mathrm{r}=0.225$ ), and conscientiousness (Sig. $=0.041$; $\mathrm{r}=0.144$ ). But it had no significant relationship with extraversion ( $\mathrm{Sig} .=0.185 ; \mathrm{r}=0.094$ ), agreeableness ( $\mathrm{Sig} .=0.921$; $\mathrm{r}=-0.007$ ), and alexithymia (Sig. $=0.058 ; \mathrm{r}=0.134$ ). Personality traits had a significant negative relationship with alexithymia (Sig. $=0.000 ; \mathrm{r}=0.523$ ) and $\mathrm{OCD}(\mathrm{Sig} .=0.000$; $\mathrm{r}=-0.253$ ). Alexithymia had a significant positive relationship with OCD (Sig. $=0.000 ; \mathrm{r}=0.272)$.

For performing SEM in AMOS software, the initial measurement model was examined by confirmatory fac-

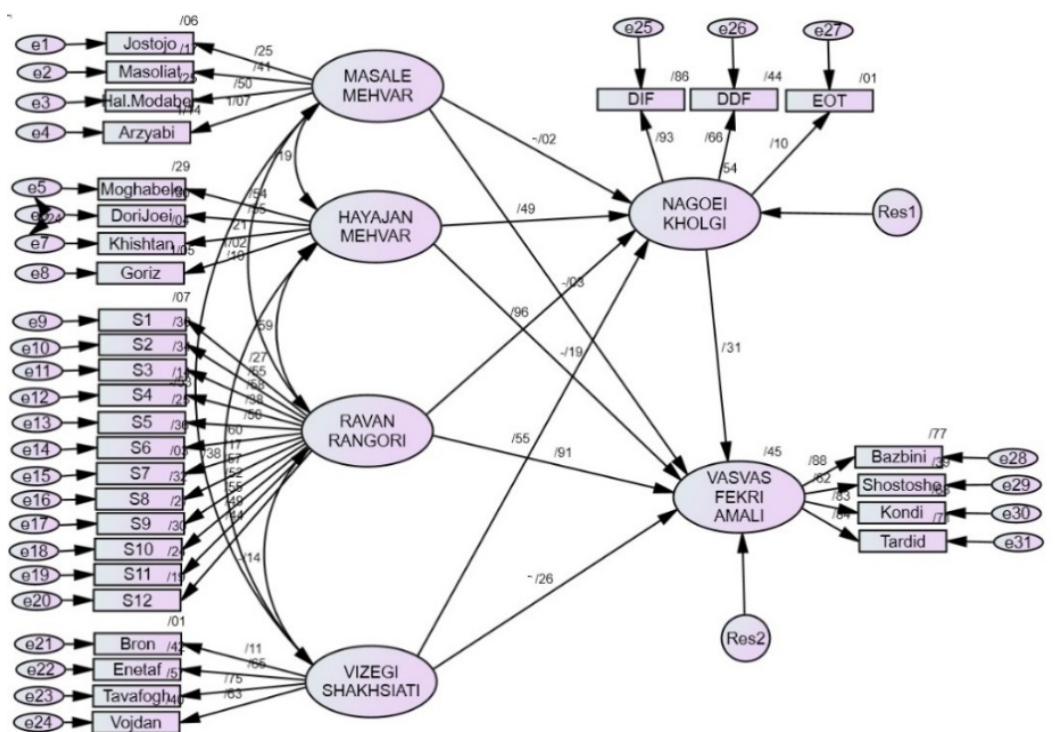

Figure 1. Structural equation modeling of the study and their path coefficients 
Table 2. The Pearson Correlation test results

\begin{tabular}{|c|c|c|c|c|c|c|c|c|c|}
\hline No. & Variables & 1 & 2 & 3 & 4 & 5 & 6 & 7 & 8 \\
\hline 1 & Problem-focused coping & 1 & & & & & & & \\
\hline 2 & Emotion-focused coping & $-0.375^{* *}$ & 1 & & & & & & \\
\hline 3 & Neuroticism & $-0.206^{* *}$ & $-0.453^{* *}$ & 1 & & & & & \\
\hline 4 & Extraversion & 0.061 & 0.094 & $-0.355^{* *}$ & 1 & & & & \\
\hline 5 & Openness to experience & 0.063 & 0.225 & $-0.258 * *$ & -0.054 & 1 & & & \\
\hline 6 & Agreeableness & -0.085 & 0.007 & 0.325 & $-0.272^{* *}$ & $-0.446 * *$ & 1 & & \\
\hline 7 & Conscientiousness & $-0.233^{* *}$ & 0.144 & -0.103 & 0.177 & -0.174 & $0.512^{* *}$ & 1 & \\
\hline 8 & Alexithymia & $-0.275^{* *}$ & 0.134 & $0.377^{* *}$ & $0.309 * *$ & 0.071 & 0.124 & $0.461^{* *}$ & 1 \\
\hline 9 & $\begin{array}{l}\text { Obsessive-compulsive } \\
\text { disorder }\end{array}$ & -0.045 & $0.198 * *$ & $0.424 * *$ & $-0.339 * *$ & 0.093 & -0.180 & 0.146 & $0.272^{* *}$ \\
\hline
\end{tabular}

Table 3. The acceptable and reported value of goodness of fit indexes for the measurement model

\begin{tabular}{|c|c|c|c|}
\hline Reported Value & Result & Acceptable Value & Index \\
\hline 2.174 & Confirmed & 3> & $\chi^{2} / \mathrm{df}$ \\
\hline 0.075 & Confirmed & $0.08>$ & RMSEA \\
\hline 0.73 & Confirmed & $0.5<$ & PNFI \\
\hline 0.63 & Rejected & $0.8<$ & GFI \\
\hline 0.57 & Rejected & $0.8<$ & AGFI \\
\hline 0.91 & Confirmed & $0.9<$ & $\mathrm{NFI}$ \\
\hline 0.94 & Confirmed & $0.9<$ & NNFI \\
\hline 0.95 & Confirmed & $0.9<$ & CFI \\
\hline 0.89 & Rejected & $0.9<$ & RFI \\
\hline 0.95 & Confirmed & $0.9<$ & IFI \\
\hline
\end{tabular}

tor analysis, whose results showed that the factor load of items was more than 0.3 and the significance level was $>$ 1.96. (items with low factor load were removed from the model, and the model was re-examined) Then, in evaluating the structural model, the conceptual model of the study and hypotheses were examined (Figure 1). In assessing the goodness of fit of study model, $\chi^{2} / \mathrm{df}$ value should be less than 3 ; RMSEA $<0.08$; PNFI $>0.05$; GFI and AGFI $>0.8$; and NFI, NNFI, GFI, RFI and IFI values $>0.9$. According to Table 3 , the values of GFI, CFI, and AGFI were more than $0.9, \chi^{2} / \mathrm{df}=2.174$, and $\mathrm{RMSEA}=0.075$. Accord- ing to these findings, the data are statistically compatible with the factor structure and theoretical basis; therefore, the measurement model has a good fit.

\section{Discussion}

The purpose of this study was to investigate the mediating role of alexithymia in the relationship between coping strategies and personality traits with OCD. The findings showed that the relationship between coping styles and OCD is significant through alexithymia. This finding is consistent with 
the results of Habibi et al. [47], De Berardis et al. [48], Kang et al. [49], and Davis and Humphrey [50]. Davis and Humphrey found that people who cannot understand and regulate their emotions have poor emotion-focused coping skills in response to stressful situations, making them more prone to mental disorders. However, people with higher levels of emotion perception ability use effective coping strategies to regulate their emotions and protect themselves from psychological problems [50]. People with OCD have concrete and externally-oriented thinking and lack the power to think about internal events. Hence, they continuously try to suppress these thoughts and their associated emotions. As a result, these suppressed thoughts and feelings turn into mental rumination. De Berardis et al. [48] found that alexithymia in OCD patients leads to emotional arousal and interferes with life's stresses. The obsessive thoughts are severe and upset the patients so much that they are forced to have obsessive behaviors and thoughts to reduce the pressure. To control these thoughts, they need the ability to cope with this pressure. Increasing the use of coping strategies increases people's ability to deal with psychological problems.

Increased use of emotion-focused coping strategy corresponds to increased anxiety, bodily harm, and obsession, while decreased use of problem-focused coping strategy corresponds to increased social dysfunction. Thus, the emotion-focused skill is more effective on physical and emotional symptoms, while the problem-focused skill is more effective on individual interactions and social functioning [48]. Lazarus and Folkman [27] stated that if people find stressors to be manageable, they are more likely to use problem-focused coping. Otherwise, they are more likely to engage in an emotion-focused coping style. This condition may explain why problem-focused coping is often used in relation to work-related stressors and social interactions, and emotion-focused coping is often used in relation to health-related stressors and emotional states. In coping processes, cognitive skills are used to solve the problem. Individuals use cognitive skills to solve problems by applying an effective problem-focused coping strategy, which leads to their psychological satisfaction.

On the other hand, this situation causes mental wellbeing and cohesion and reduces emotional turmoil. The source of obsession is identified better and can be assessed as controllable because of the obtained mental cohesion and wellbeing. In other words, it helps to increase mental health. Low levels of stress make it easier for a person to use psychological skills to cope with problems with peace of mind. Overall, emotionfocused coping skill controls the emotional consequences of a stressful event and maintains emotional balance by controlling the emotions resulted from a stressful situation.
In contrast, problem-focused coping, which is related to the individual's effective actions in stressful situations, causes the source of stress to be removed or changed. Therefore, this issue can directly affect OCD or indirectly by affecting alexithymia. In other words, considering that alexithymia has a significant positive correlation with anxiety, it can improve or worsen OCD. However, more study is needed in this area to achieve detailed results.

This study showed that the relationship between personality traits and OCD was significant after mediation by alexithymia. This finding is consistent with the results of De Berardis et al. [48], Kang et al. [49], Khosravani et al. [51], and Piri et al. [52]. High harm avoidance in the biopsychosocial model of Robert Cloninger et al. [53], low sensation seeking in Zuckerman's alternative five-factor model of personality, and the role of thoughts in creating and sustaining obsessions and compulsions have also shown this vulnerability. Studies on the five-factor personality model also found that people with OCD had high neuroticism scores and low scores in extraversion and conscientiousness. However, in openness to experience and agreeability, some studies have shown average scores. Higher scores on neurotic characteristics are associated with a greater willingness to experience negative emotions and increased psychological problems [54]. Neuroticism is one of the personality traits that is opposed to emotional stability. People with OCD are more introverted and dogmatic and experience more negative emotions, such as anxiety and depression [54]. In the present study, no relationship was found between the extraversion trait and OCD. In other words, extroverted people are less likely to develop OCD. Extroverts can talk about their problems with others because of having proper communication with others in stressful situations. This skill relieves their pressure. In contrast, introverts in stressful situations can be catastrophic, and their poor communication causes them to use obsessive-compulsive behaviors as a way to communicate with others instead of expressing their psychological distress.

The present study had some limitations related to the accuracy and generalizability of the results. One of the limitations was using a self-report tool, and the questionnaires may not be answered accurately. This study was performed on OCD patients in Kerman City, and therefore we should be cautious about the generalizability of the results. The limitation of the statistical population to OCD patients and the lack of consideration of some confounding variables such as economic, social, cultural, and family status, limit the generalizability of the above findings. Therapists and counselors who deal with people with OCD are recommended to pay attention to personality traits and mental disorders associated with the disease 
because knowing these variables can help provide better and more appropriate services to these patients.

\section{Conclusion}

Modeling the relationship between coping strategies and personality traits with OCD should be based on the mediating role of alexithymia. It plays an essential role in improving the mental health of OCD patients.

\section{Ethical Considerations}

\section{Compliance with ethical guidelines}

All ethical principles are considered in this article. The participants were informed of the purpose of the research and its implementation stages. They were also assured about the confidentiality of their information and were free to leave the study whenever they wished, and if desired, the research results would be available to them (Code: IR.KMU.REC.1398.527).

\section{Funding}

This article is extracted from the $\mathrm{PhD}$. dissertation of Maryam Pour Amrollahi's thesis in General Psychology at Bahonar University, Faculty of Literature and Humanities.

\section{Authors' contributions}

All authors equally contributed to preparing this article

\section{Conflicts of interest}

The authors declared no conflict of interest

\section{Acknowledgements}

The authors would like to thank the Deputy for Research of Islamic Azad University of Zarand branch and Kerman University of Medical Sciences. 
This Page Intentionally Left Blank 


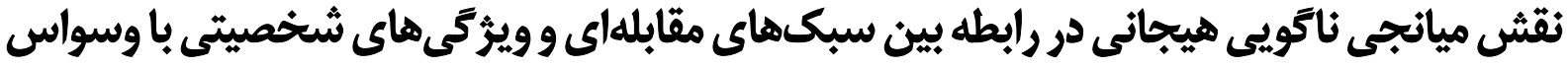 فكرى عملى فئس
}

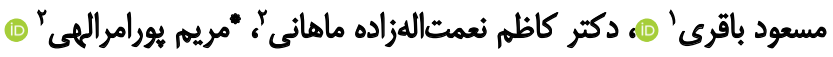 \\ 1. كروه روائشناسي، دانشكده ادبيات و علوم انساني، دانشكاه شهيد باهنر كرمانء ايران.

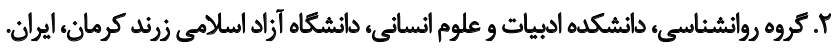

\begin{abstract}
Q

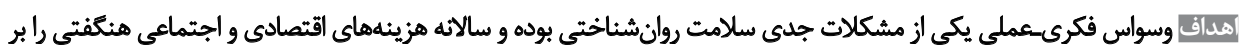

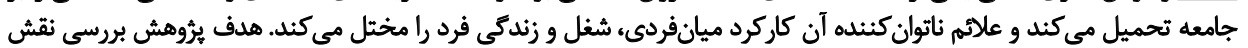

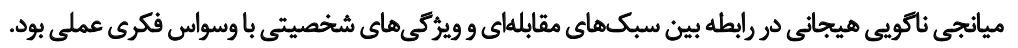

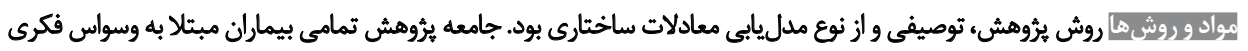

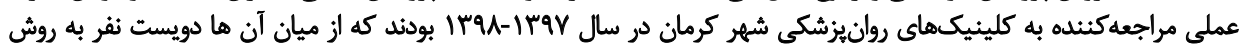

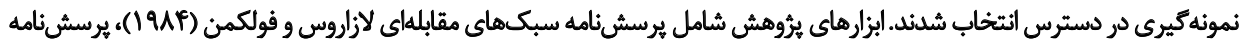

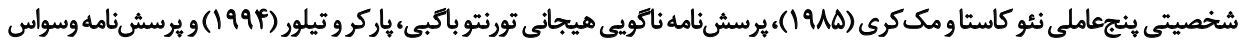

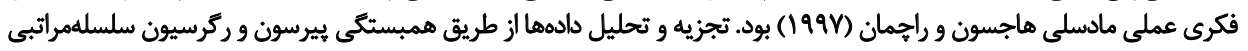

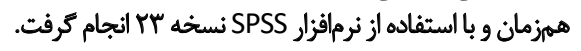

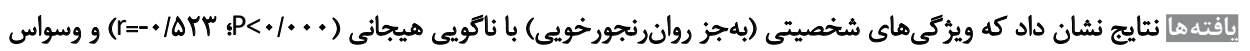

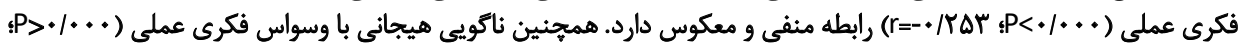

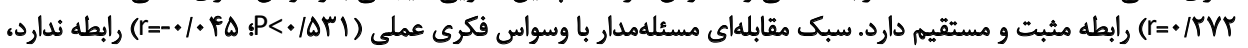

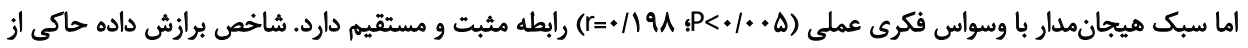

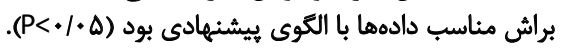

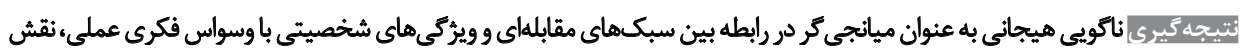

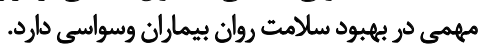

تاريخ دريافت: 11 اسفند

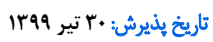

تاريخ انتشار: rادى

\section{: Lojlgh \\ ناكويى هيجاني، مقابله، \\ شخصيت، وسواس.}

اختلال وسواس فكرى عملى' يكى از متداولترين اختلالات

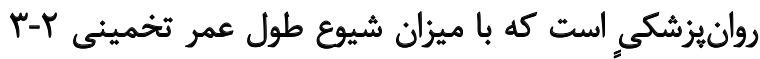

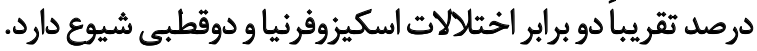

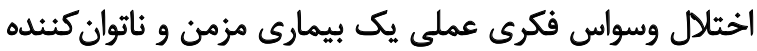

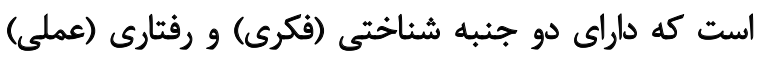

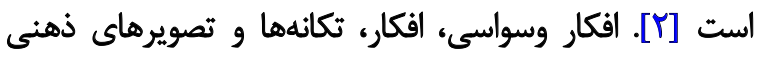

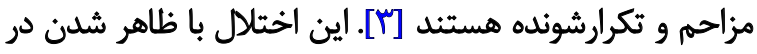

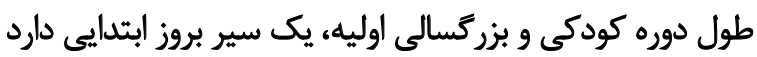

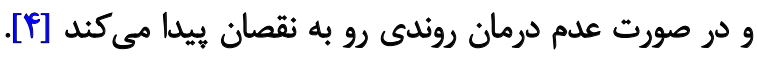

1. Obsessive Compulsive Disorder(OCD) doleo در دنياى يُيجيده امروز، انسان با مسائل و مشكلات زيادى إنى

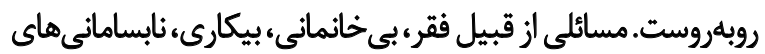

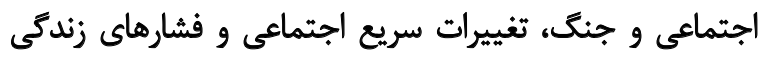

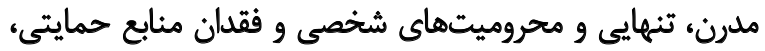

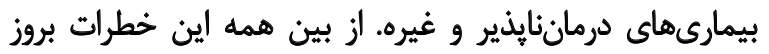

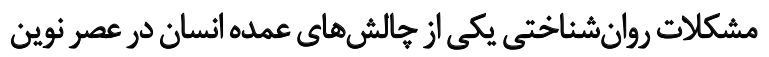

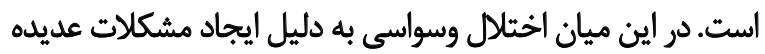

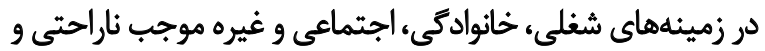

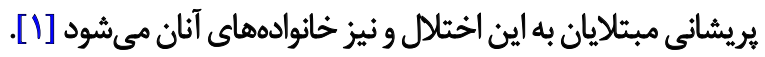

\section{a.}

* نويسنده مسئول:

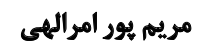
نشائي: كرمان، دانشعاه آزاد اسلامى زرند، دانشكده ادبيات و علوم انسانى، كروه روانشناسى. تلفن: بست الكترونيكي: mashizi88@yahoo.com 
وجود داشت. نهايتاً سازكارى در هر دو گروه با با وجدان بودن

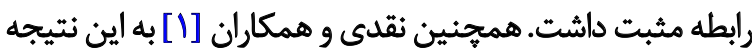

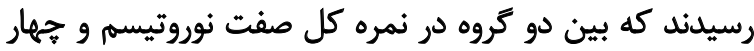

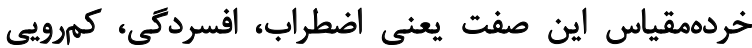

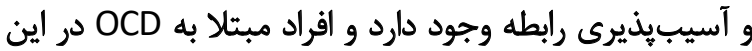

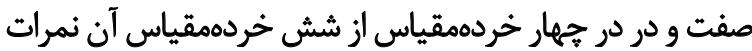

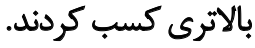

به نظر مىرسد كه سبكهاى مقابلهاي" با وسواس مرتبط

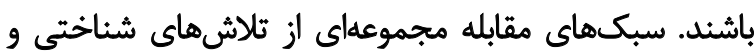

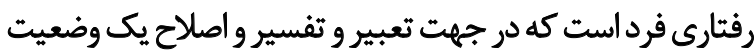

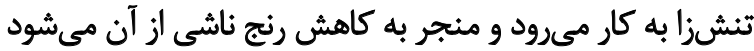

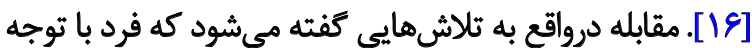

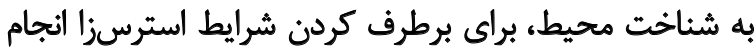

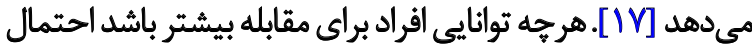

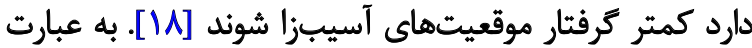

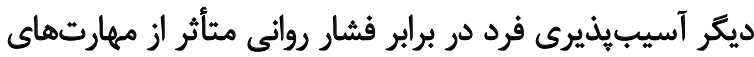

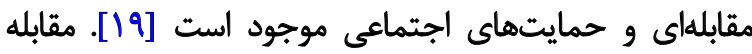

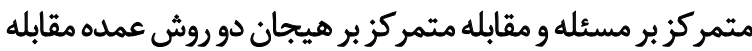

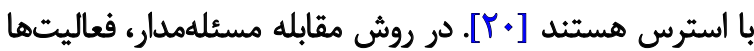

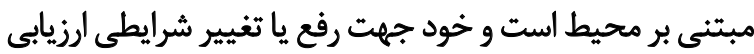

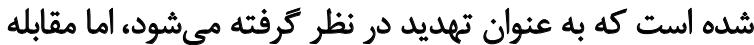

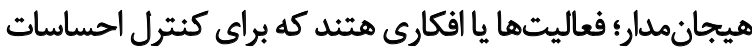

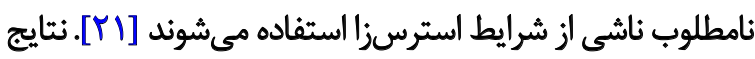

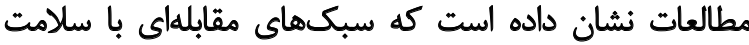

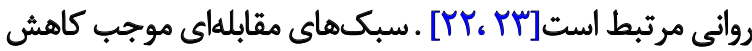

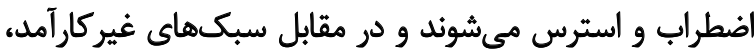

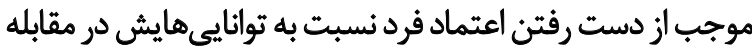

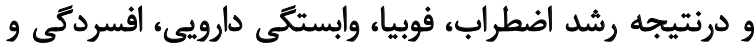

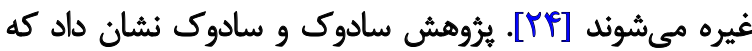

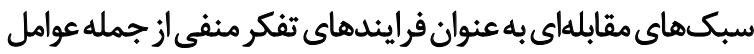

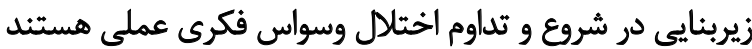

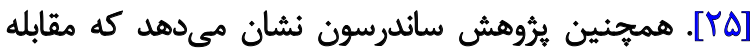

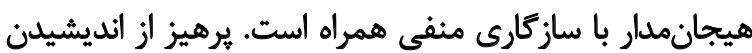

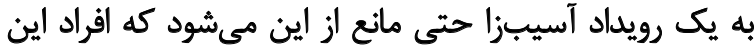

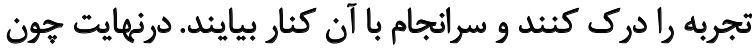

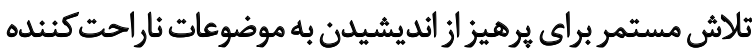

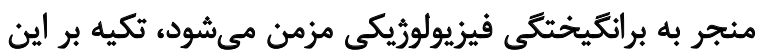

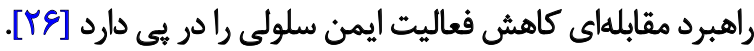

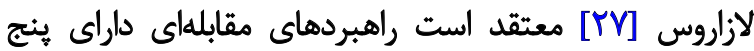

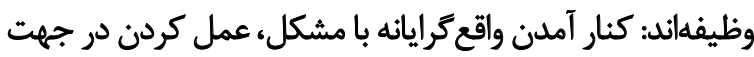

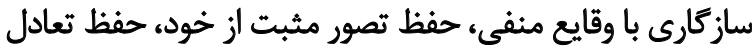

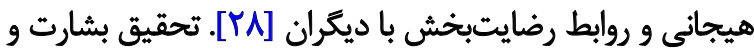

9. Coping strategies
راهنماى تشخيص آمارى اختلالهاى روانى' [هـ] بيان مىكند

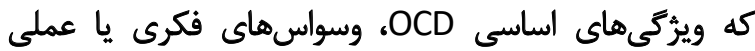

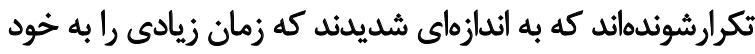

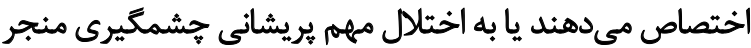

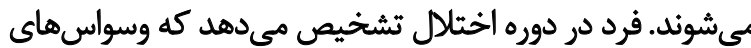

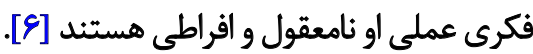
يكى از متغيرهايى كه با وسواس ارتباط دارد، ويرّكى هاى

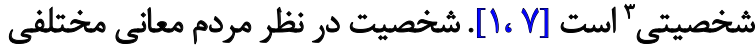

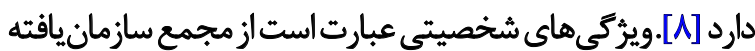

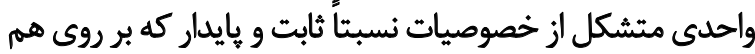

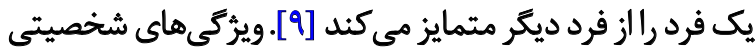

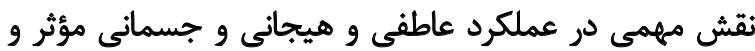

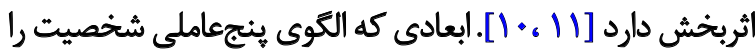

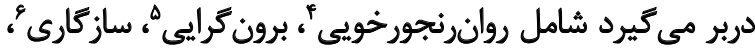

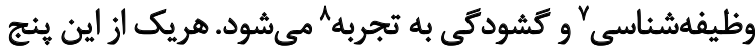

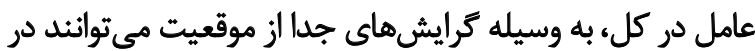

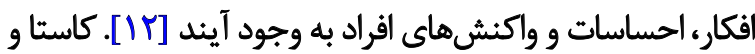

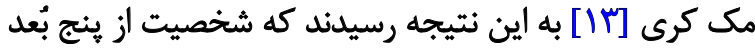

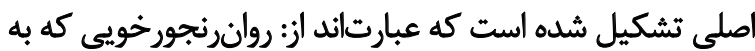

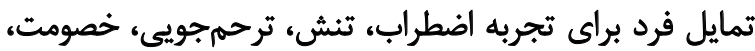

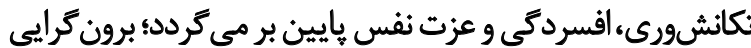

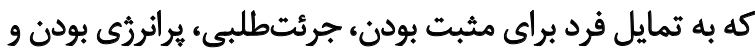

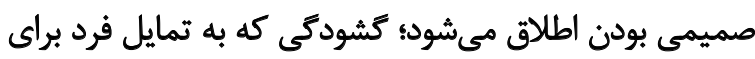

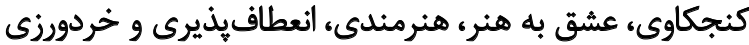

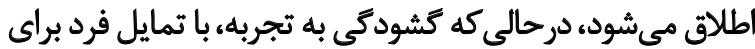

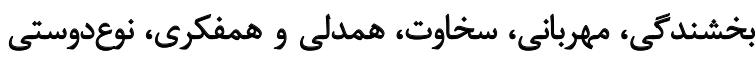

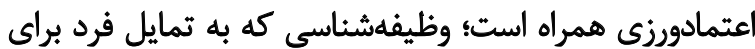

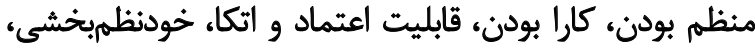

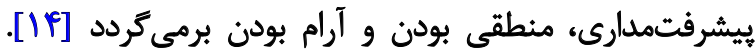

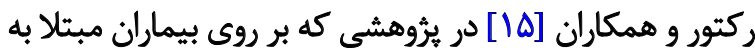

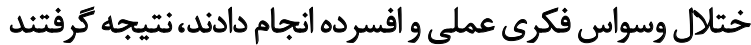

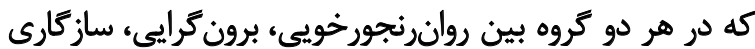

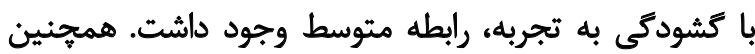

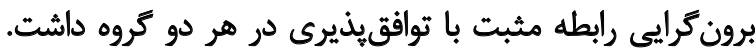

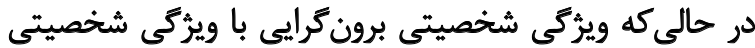

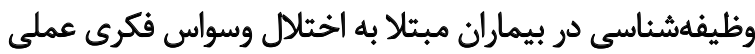
رابطه نداشت، ولى رابطه مثبتى بين اين ابعاد در كروه افسرده

2. Diagnostic and Statistical Manual of Psychiatric Disorders 3. Personality characteristics

4. Neuroticism

5. Extraversion

6. Agreeableness

7. Conscientiousness

8. Openness to Experience 
را بيشتر مى كند. بررسى ارتباط نشائكان وسواس فكرى عملى بائ

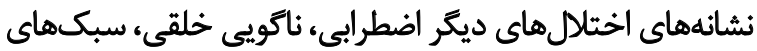

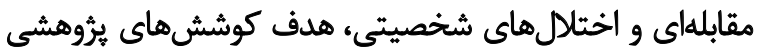

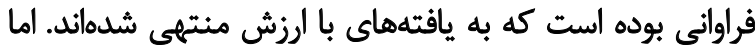

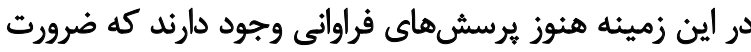

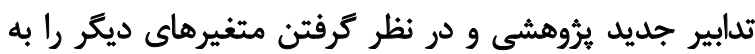

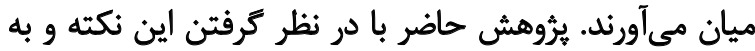

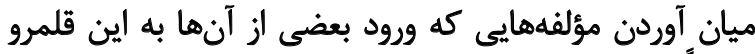

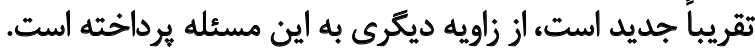

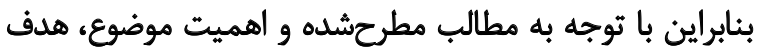
اصلى مطالعه حاضر نقش ميانجى ناكويى هيجانى در در رابطه بين بين

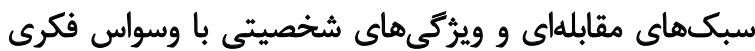

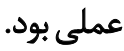

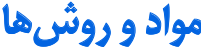

روش يُروهش حاضر، توصيفى و از نوع مدل يابى معادلات

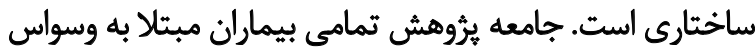

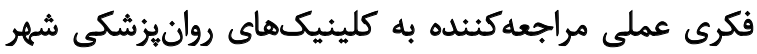

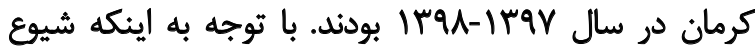

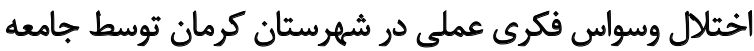

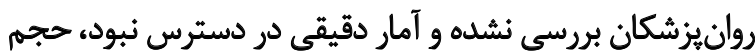

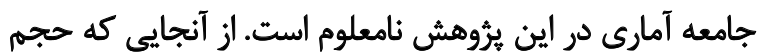

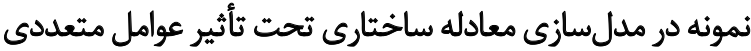

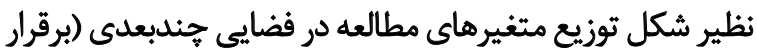

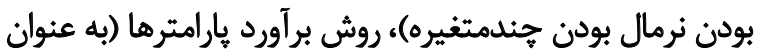

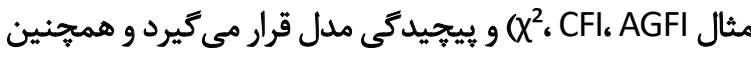

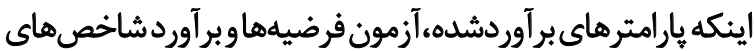

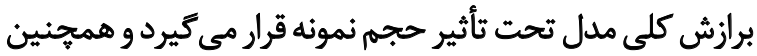

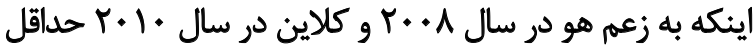

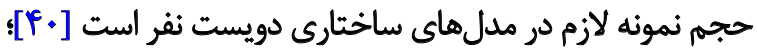

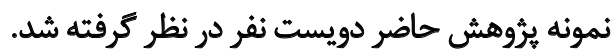

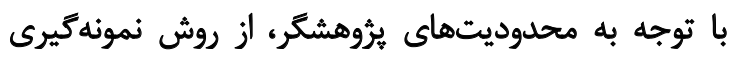

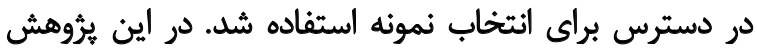

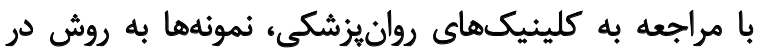

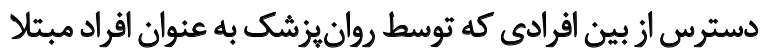

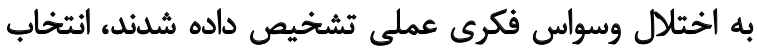

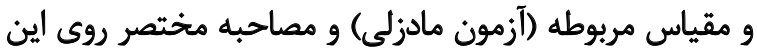

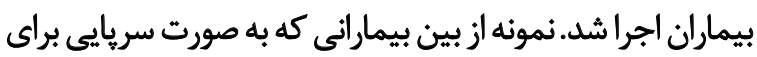

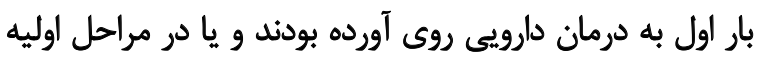
درمان قرار داشتند، انتخاب شدند.

بعد از مشخص شدن دقيق نوع بيمارى آزمودنى ها، دستور العمل

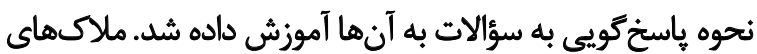

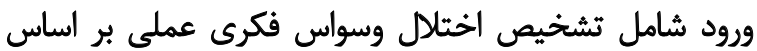

همكاران [Y]] با هدف تبيين سلامت روانى بر حسب سبكهاي

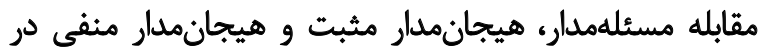

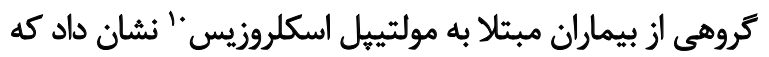

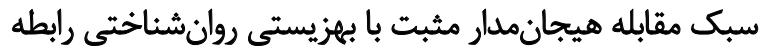

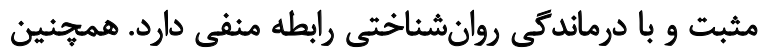

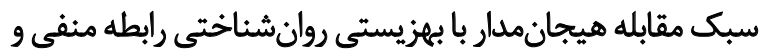

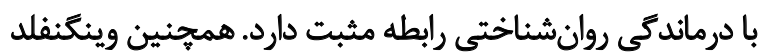

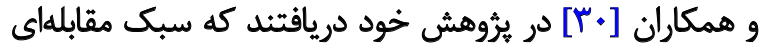

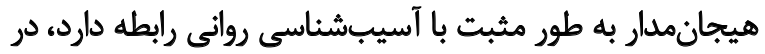

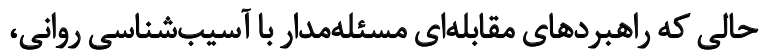

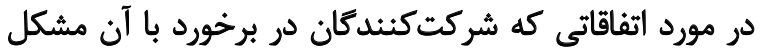
داشتند، همبستكى منفى نشان داد.

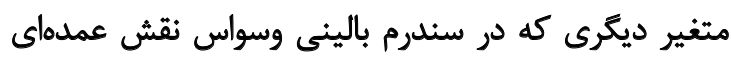

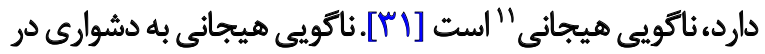

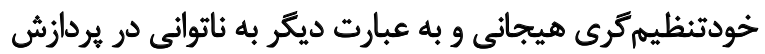

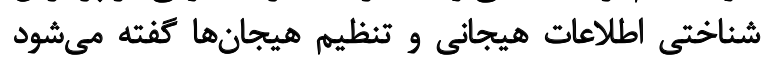

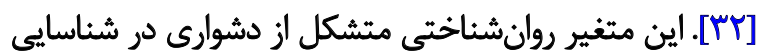

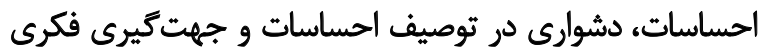

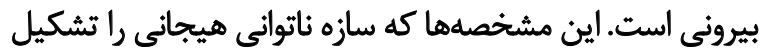

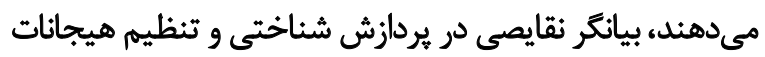

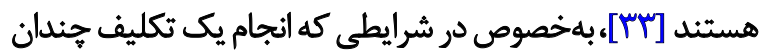

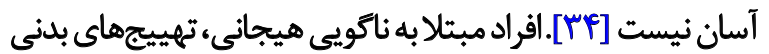

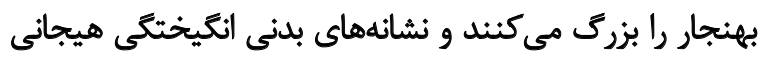

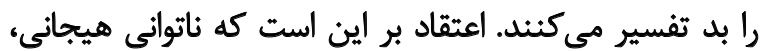

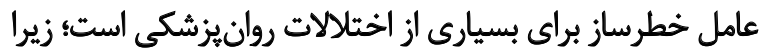

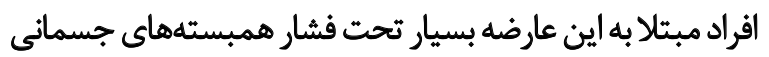

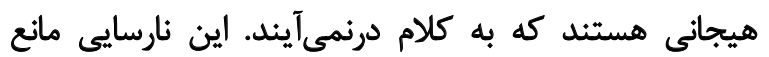

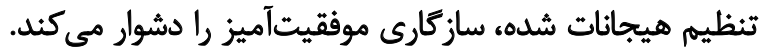

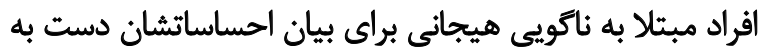

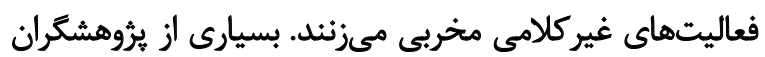

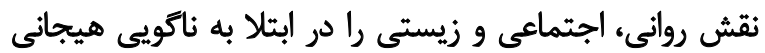

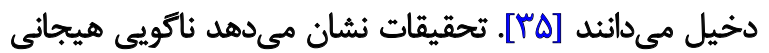

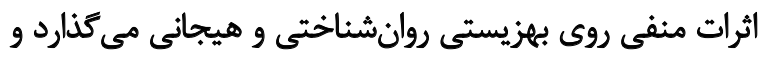

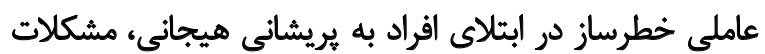

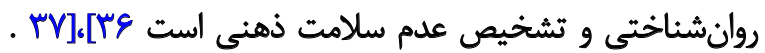

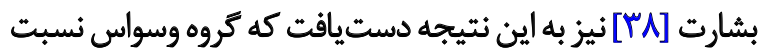

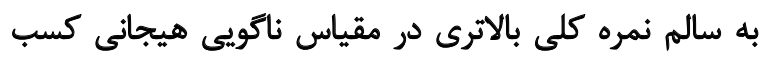

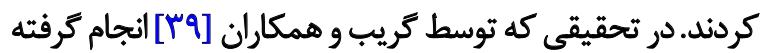

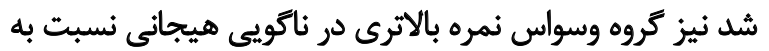

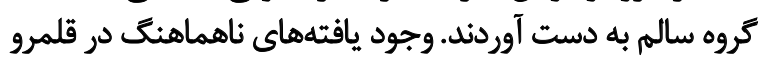

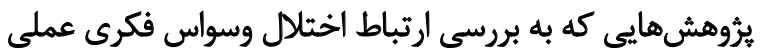

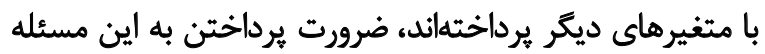

10. Sclerosis (MS)

11. Alexithymia 


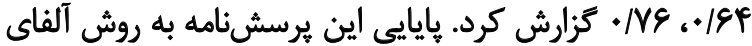

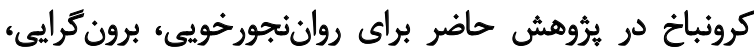

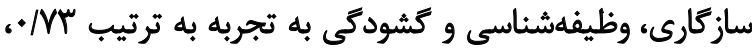
年

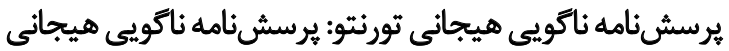

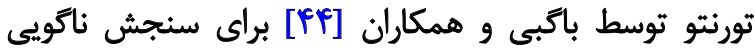

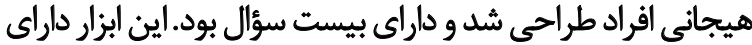

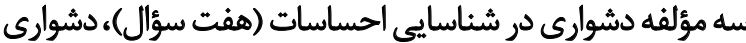
در توصيف احساسات (ينج سؤال) و تفكر عينى (هشت سؤروال)

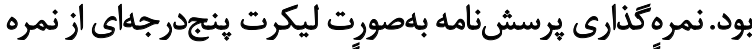

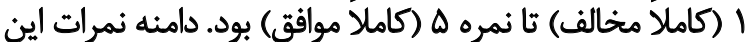

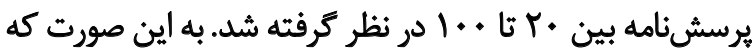

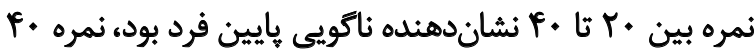

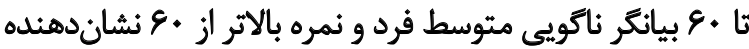

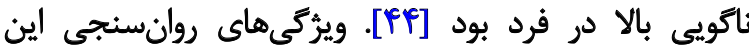

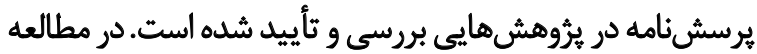

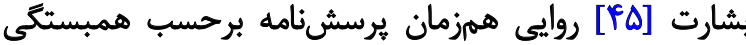

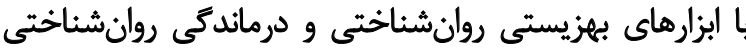

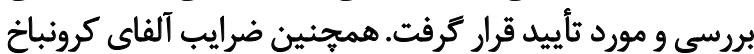

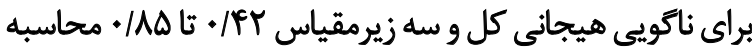

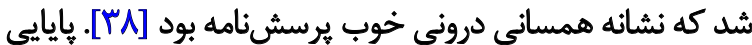

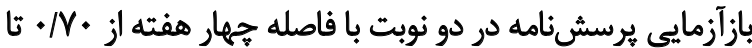

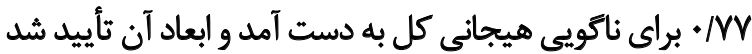

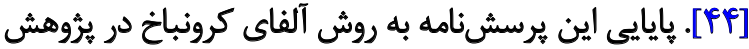

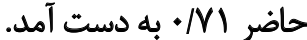

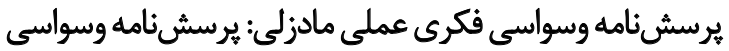

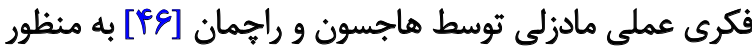

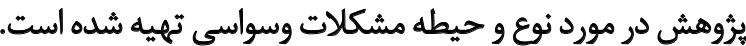

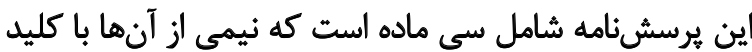

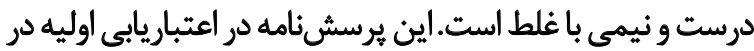

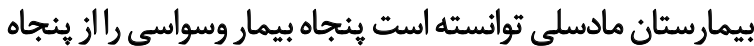

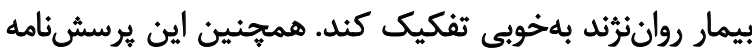

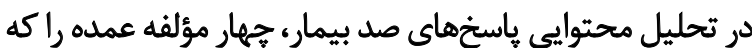

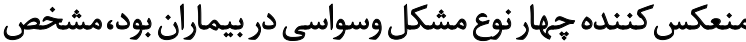

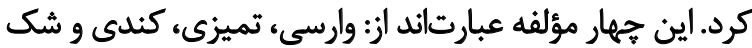

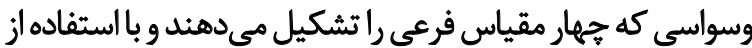

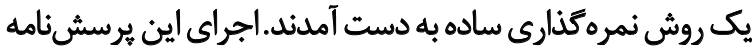

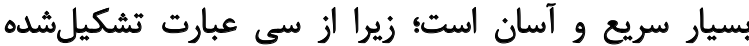

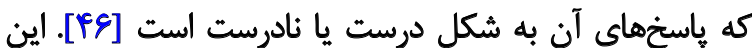

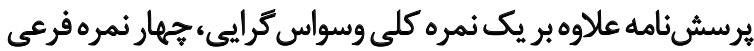

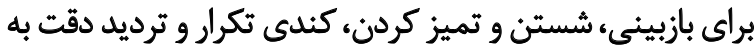

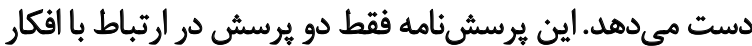

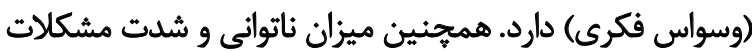

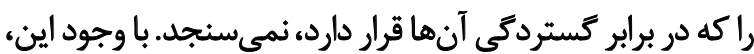

مصاحبه بالينى و تأييد تشخيص بر اساس نظر روانيزشك،

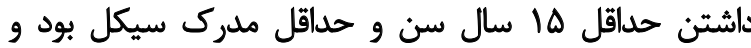
ملاكهاي خروج هم شامل اختلالات سايكوتيك، اختلال توره،

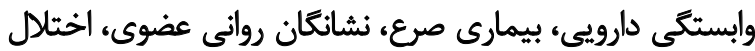

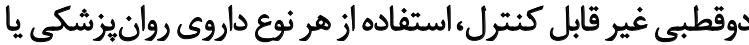

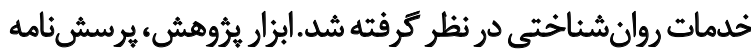

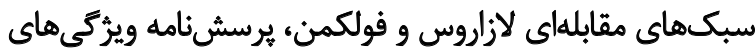

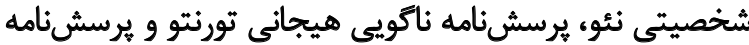
وسواسى فكرى عملى مادسلى بود.

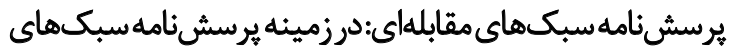

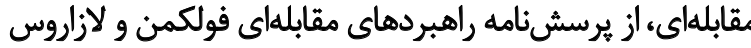

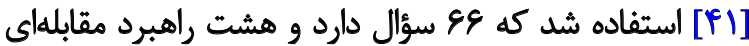

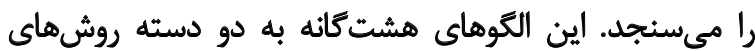

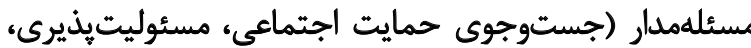
حل مدبرانه مسئله و ارزيابى مجدد مثايت مثبت) و هيجاعي، مئنمدار (مقابله

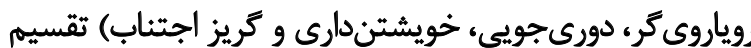

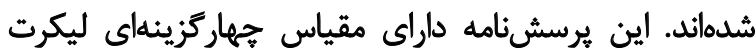

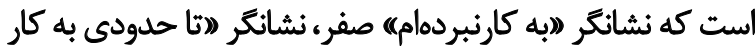

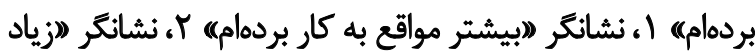

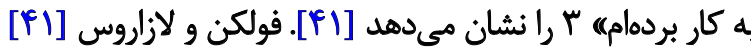

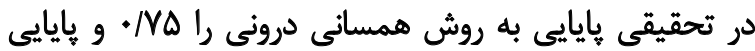

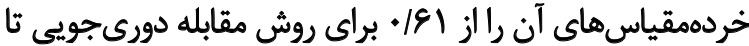

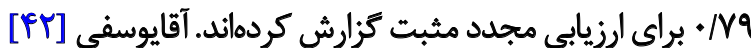

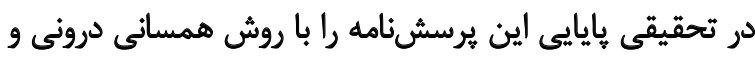

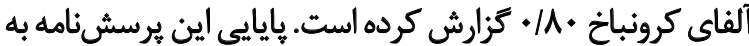

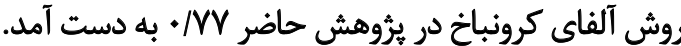

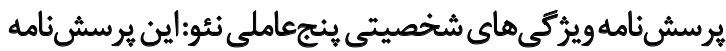

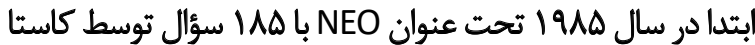

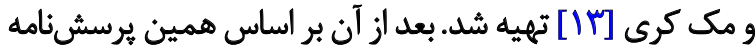

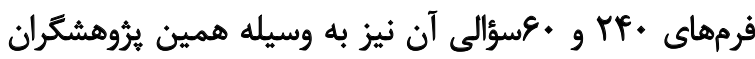

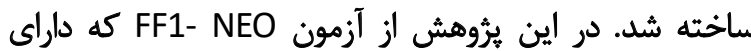

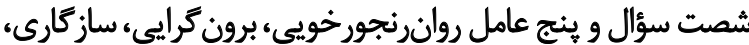

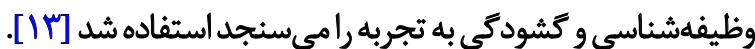

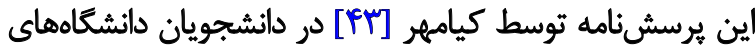

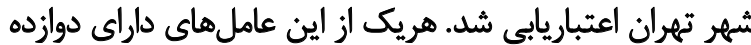

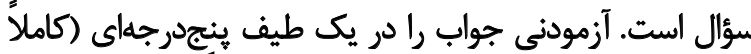

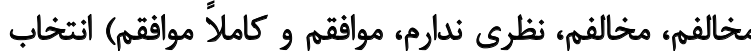

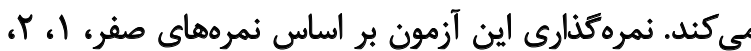

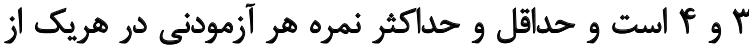

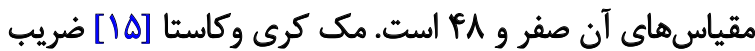

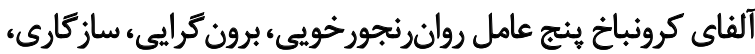

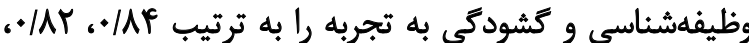

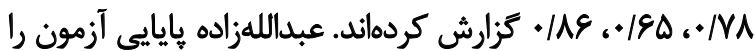

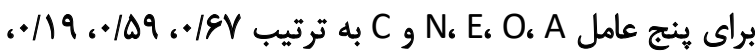




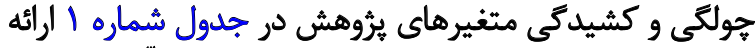

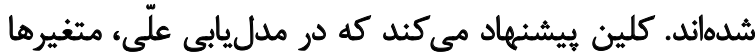

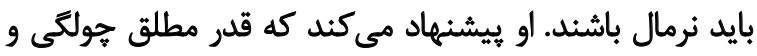

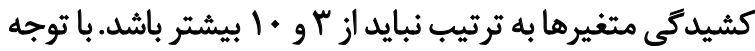

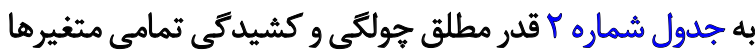

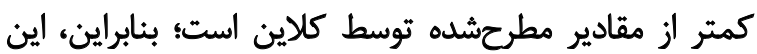

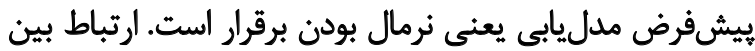

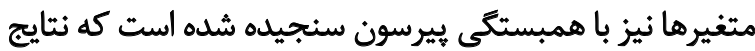
در جدول شماره ب كزارش شده است.

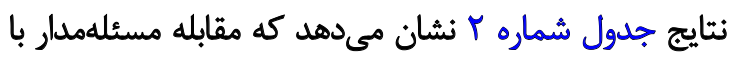

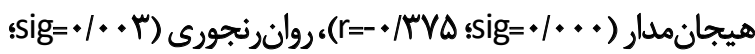
خ

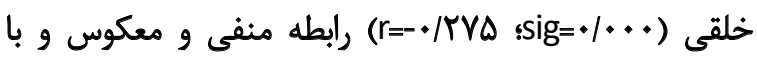

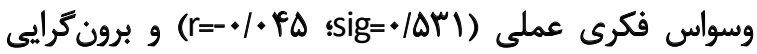

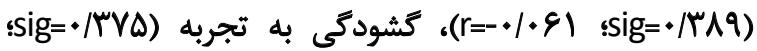
مقابله

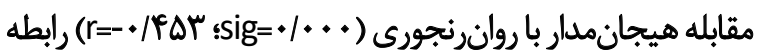

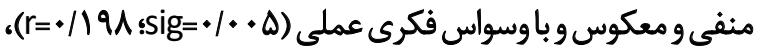

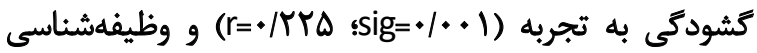

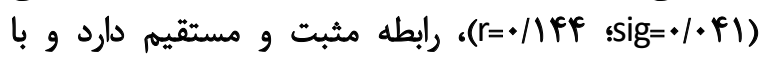

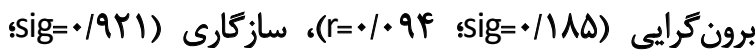

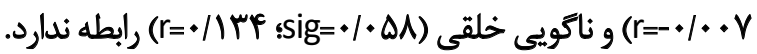

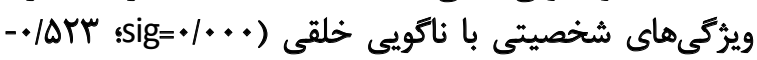

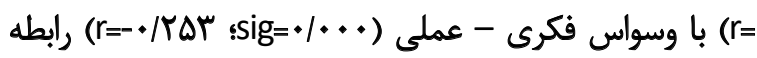

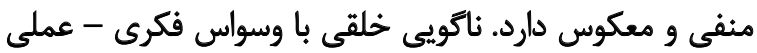

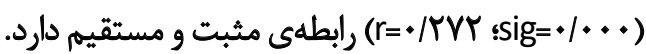

در اين برثوهش از مدلسازى معادلات ساختارى استفاده

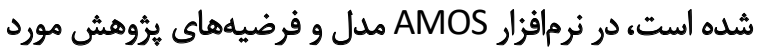

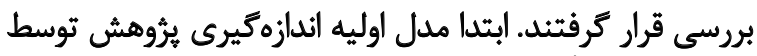

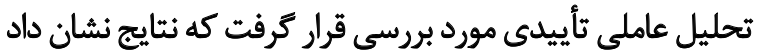

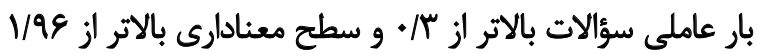

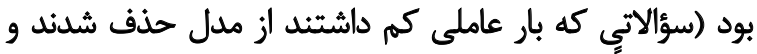

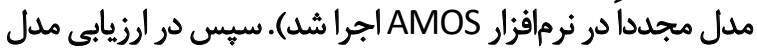

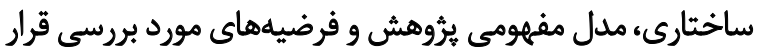

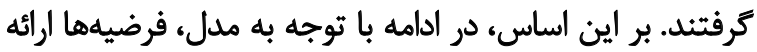

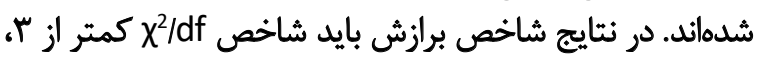

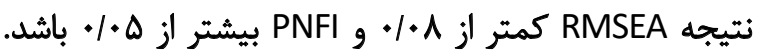

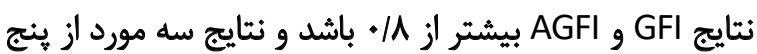

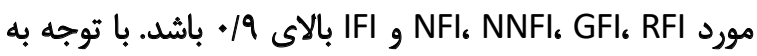

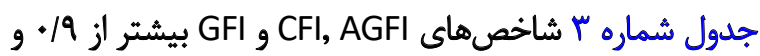

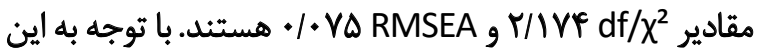

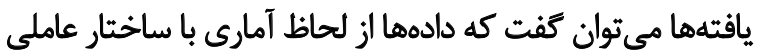

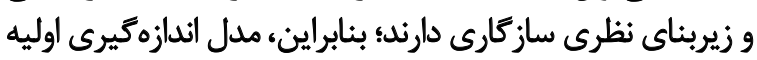

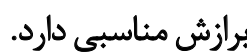

مى تواند بيماران مبتلا به وسواس فكرى عملى را الز ساير اشخاص

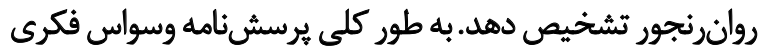

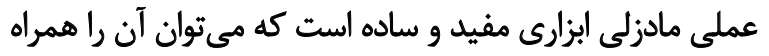

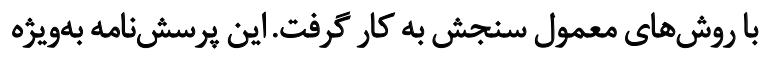

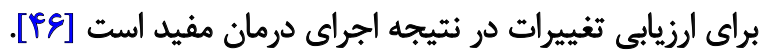

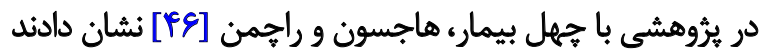

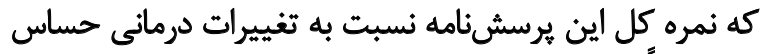

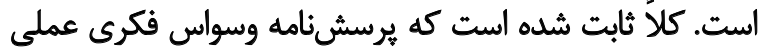

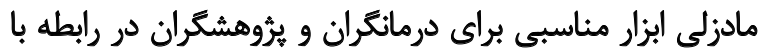

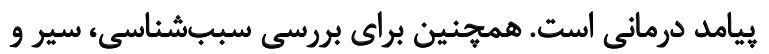

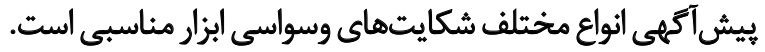

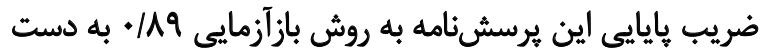

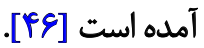

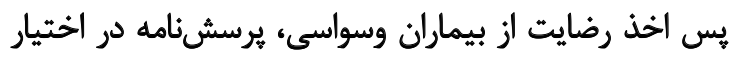

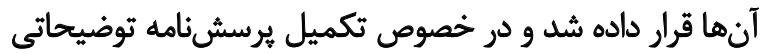

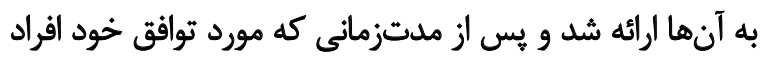

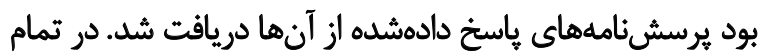

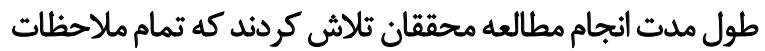

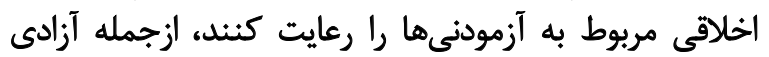

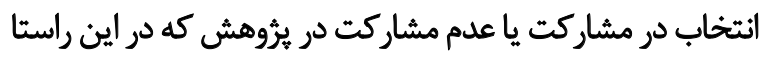

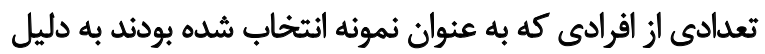

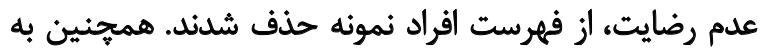

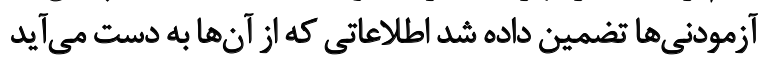

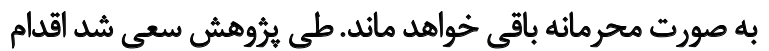

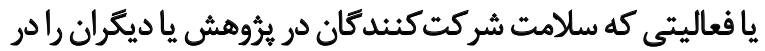
معرض خطر قرار دهد، انجام نكيرد.

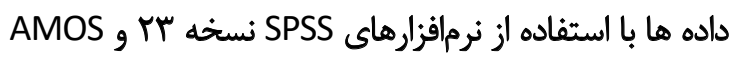

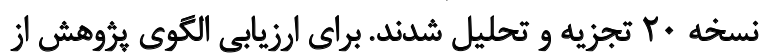

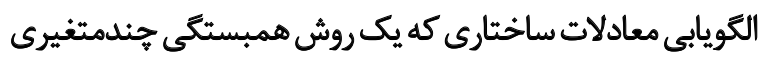
الست، استفاده شد.

يافتهها

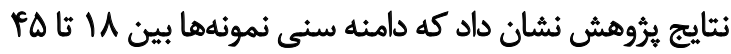

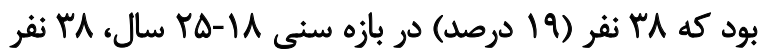

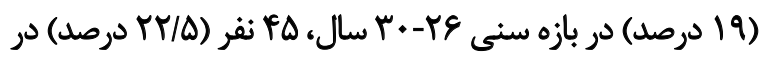

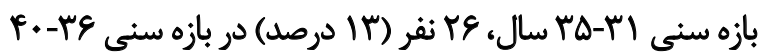

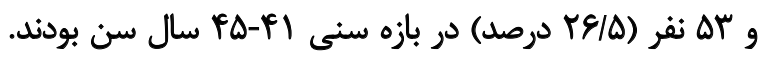

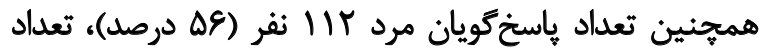

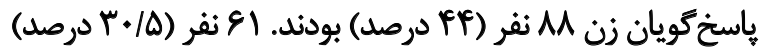

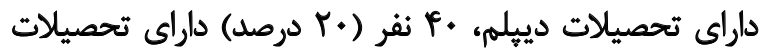

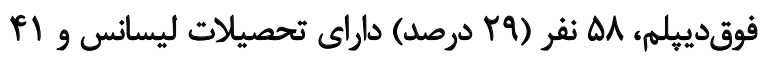

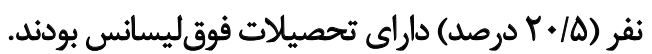
يافتههاى توصيفى ميانتين،انحراف معيار،كمترينو بيشترين. 
جدول ا. وضعيت توصيفى متغيرهاى بثروهش

\begin{tabular}{|c|c|c|c|c|c|c|}
\hline كشيدكى & جولكىى & بيشترين & كمترين & ميانكين 土 انحراف معيار & 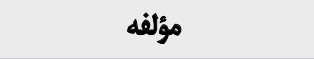 & مثغير \\
\hline$-/ 191$ & .1119 & IV & r & $q / v \Delta \Delta \pm r / \cdot 1 r$ & جستوجوى حمايت اجتماعى & \multirow{5}{*}{ مسئلمملار } \\
\hline . TYA & $\cdot / r+1$ & ir & $r$ & $V / r V \cdot \pm Y / \cdot \Delta)$ & مسئوليتبذيرى & \\
\hline$-* / r i f$ &.$- / 199$ & 10 & r & $N T \Lambda \Delta \pm Y M r$. & حل مدبرائه مسئله & \\
\hline.$- / r \cdot 4$ &.$/ 1 W$ & r. & r & $\mid r / \cdot f \Delta \pm \Psi / M V$ & الرزيابى هجدد مثبت & \\
\hline.$/ N \Delta \Delta$ & .1918 & $\Delta f$ & Tr & $r V / N \Delta \Delta \pm \Delta / \mathscr{R T}$ & 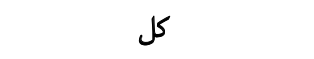 & \\
\hline - - & $-\pi r$ & if & r & $N T V \cdot \pm T / M T A$ & مقابله روياروكر & \multirow{5}{*}{ هيجانمدار } \\
\hline $1 / 719$ & $.19 W$ & iv & $r$ & Q/ITLIT/PAT & دورجويى & \\
\hline$\% \Delta r$ & 吕 & 19 & 8 & $W / \tau+ \pm t r / V \cdot Y$ & خويشتندارى & \\
\hline$\cdot / 1 \cdot \Delta$ & | & rt & $f$ & $1 . / Q \varepsilon . \pm N / N \Delta S$ & كريز اجتناب & \\
\hline $\mathrm{N} \cdot \mathrm{Ir}$ &.$/ r A 1$ & 94 & $M$ & 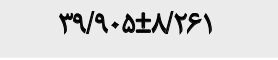 & كل & \\
\hline$-+/ M+1$ & $\mathscr{~} / \cdot A V$ & $w$ & v & TI/NTAIV/TYA & روانرنجورى & \multirow{5}{*}{ ويثُكى هالى شخصيتى } \\
\hline$-+/$ Mr &.$/ M Y$ & $\varphi$ & 8 & $r .1 .90 \pm 9 / 199$ & 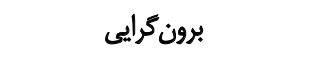 & \\
\hline.$- / f \cdot 1$ &.$- / 1 \Delta$ & $m$ & if & $T F / M 9 \Delta \pm / M q$ & كشودمى به تجربه & \\
\hline$-\cdot / \pi / \wedge$ &.$- / \cdot W$ & r. & 1. & $|2 / A 1 \cdot \pm \Psi / \Delta A|$ & سازكارى & \\
\hline$\cdot|A| A$ & $-* / \pi$ & ru & $r$ & $19 / N \Delta \cdot \pm P / 9 T V$ & وظيفهشناسى & \\
\hline .1 .10 & •/PAY & ra & r & $W Q Y \cdot \pm \Delta / \Delta \cdot r$ & دشوارى در تشخيص احساسات & \multirow{4}{*}{ ناتوبيى هيجائى } \\
\hline.$- / P T \varphi$ & $-t /$ rar & $\pi$ & $\Delta$ & $\mid r / \Delta E \Delta \pm \Psi / Q 91$ & دشوارى در توصيف احساسات & \\
\hline.$/ \Delta \mathrm{ra}$ &.$- / r \Delta r$ & $\pi$ & 1. & $r / / 90 \pm t r / .9 \Delta$ & تفكر با جهت كيرى خارجى & \\
\hline$\cdot / \mathrm{TAA}$ & $-+/ r+1$ & va & rq & $\Delta H / \varepsilon q . \pm q / \Delta l f$ & كل & \\
\hline-1919 & - MAT & 9 & • & 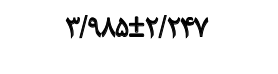 & بازيينى & \multirow{5}{*}{ وسواس فكرى عملى } \\
\hline$-+/ \Delta \& A$ & 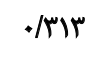 & 9 & • & $\varphi / M r+ \pm Y / 1+\varepsilon$ & مشتوشو & \\
\hline$-* / M I F$ & . Mif & 8 & • & $Y / \Delta \Delta \Delta \pm I / F \& A$ & كثنى تكرار & \\
\hline . /9Ta & . Retr & ir & 1 & $r / \cdot r \cdot \pm 1 / 1 \cdot q$ & ترديد وظيفهشناسى & \\
\hline.$- / \& \Delta S$ & 涪 & rV & $r$ & $\mid \Psi / / V \cdot \pm \Psi / T \Delta$ & كل & \\
\hline
\end{tabular}

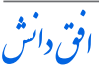

مسير غيرمستقيم مذكور مورد تأييد قرار كرفت اين يافته با نتايج

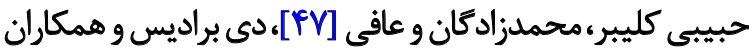

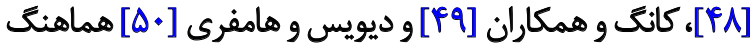

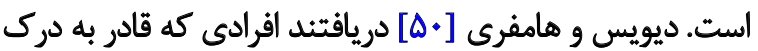

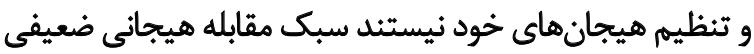

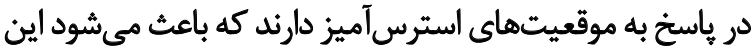

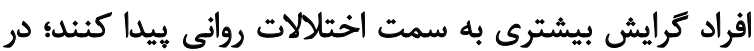

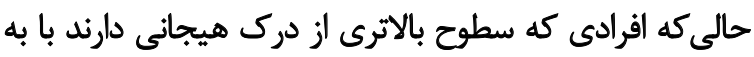

هدف از يرؤوهش حاضر، نقش ميانجى ناكويى هيجانى در رابطه

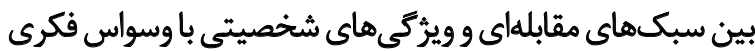

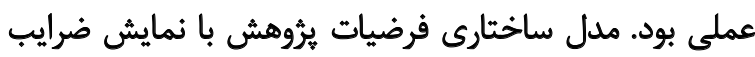

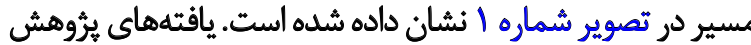

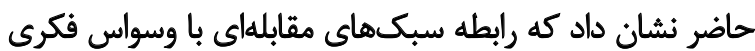
عملى از طريق ناتويى هيجانى معنى دار است؛ به به عبارت ديكرات فيكري 
جدول r. نتايج آزمون همبستكى ييرسون

\begin{tabular}{|c|c|c|c|c|c|c|c|c|c|}
\hline$\wedge$ & $\checkmark$ & 7 & • & $\varepsilon$ & $r$ & $r$ & 1 & متغير & رديف \\
\hline & & & & & & & 1 & مسئلمملار & 1 \\
\hline & & & & & & 1 &.$- / r v \Delta^{\circ}$ & هيجانمدار & $r$ \\
\hline & & & & & 1 &.$- / F \Delta r$ &.$- / r \cdot q^{*}$ & روانرنجورى & $r$ \\
\hline & & & & 1 &.$- / r \Delta \Delta^{*}$ & .1 .99 & .1 .81 & برون كرايى & f \\
\hline & & & 1 & $-.1+\Delta f$ &.$- / T A A^{*}$ &.$/ T r \Delta$ & 1.84 & كشودكى به تجربه & $ه$ \\
\hline & & 1 &.$- / 948 *$ &.$- / T r r^{*}$ &.$/ T r a$ & 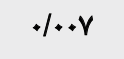 & $-.1 \cdot 1 \Delta$ & ساز كارى & 8 \\
\hline & 1 & . $/ \Delta 1 Y^{\circ}$ &.$- / 11 n$ &.$/ 1 w$ & $-* N \cdot r$ & ./IfP & -./Trm" & وظيفهشناسى & $\checkmark$ \\
\hline 1 &.$|49|^{\circ}$ &.$/ 1 Y 4$ & $\cdot / \cdot n$ &.$/ 4 \cdot 9^{\circ}$ &.$/ 4 w^{*}$ & ./Imf &.$- / T Y \Delta^{\circ}$ & ناكويى هيجانى & $\wedge$ \\
\hline.$/ r r^{*}$ &.$/ 198$ &.$- / 14$ & .1 .94 &.$- / / \% q^{*}$ & .perte* & $.114^{\circ}$ & $-.1 .+\Delta$ & وسواس فكرى عملى & 9 \\
\hline
\end{tabular}

جدول r. مقدار معيار و كزارش شده شاخصهاى برازندكى مدل اندازهيرى اوليه يثروهش

\begin{tabular}{|c|c|c|c|}
\hline شاخص & مقدار معيار & تثيجه & مقدار كزارش شده \\
\hline$\chi^{2} / d f$ & $<r$ & تأييد & r/IVP \\
\hline RMSEA & $<+1 \cdot 1$ & ثاييد & $.1 \cdot v a$ \\
\hline PNFI & $>. / \Delta$ & ثأييد &.$M$ \\
\hline GFI & $>\cdot / 1$ & د) &.$/ 8 \pi$ \\
\hline AGFI & $>\cdot / 1$ & د) &.$/ \Delta V$ \\
\hline NFI & $>. / 9$ & تأييد &.$/ 91$ \\
\hline NNFI & $>. / 9$ & تأييد &.$/ 9 f$ \\
\hline CFI & $>. / 9$ & تأييد &.$/ 90$ \\
\hline RFI & $>. / 9$ & د, &.$/ 19$ \\
\hline IFI & $>. / 9$ & تأييد &.$/ 9 \Delta$ \\
\hline
\end{tabular}

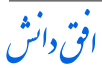

مى كند؛ به نحوى كه براى كاهش فشار افكار ناجار به انجام رفتارها

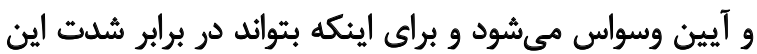

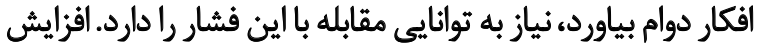

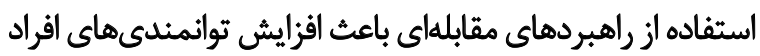

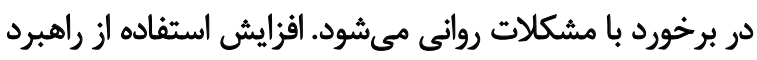

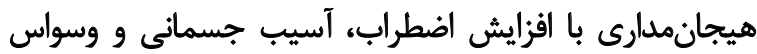

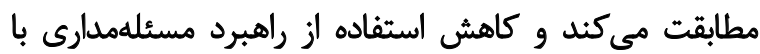

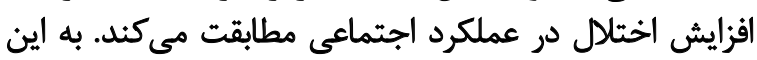

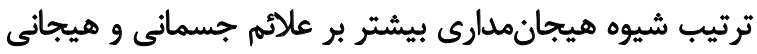
مؤثر است، درحالى شه شيوه مسئلهمدارى بيشتر بر تعاملات جيات

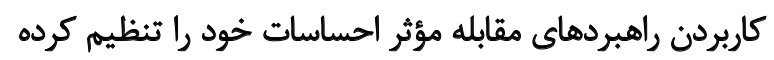

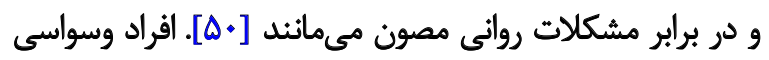

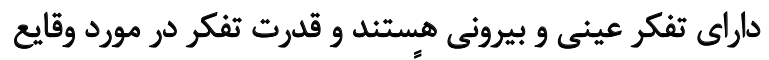

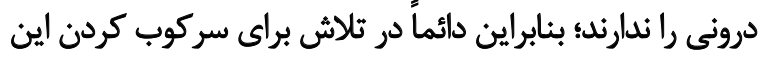

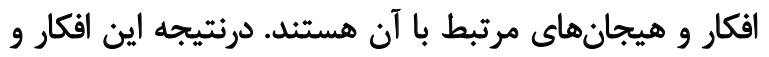

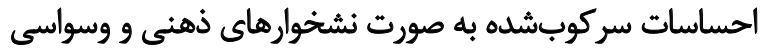

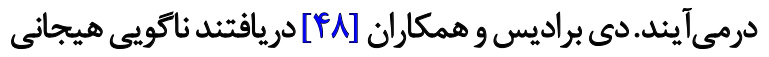

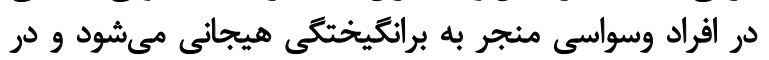

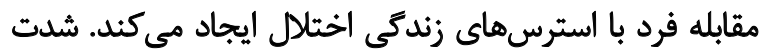
و قدرت افكار وسواسى به حدى قوى است كه بيمار إنى رابها برآشفته 


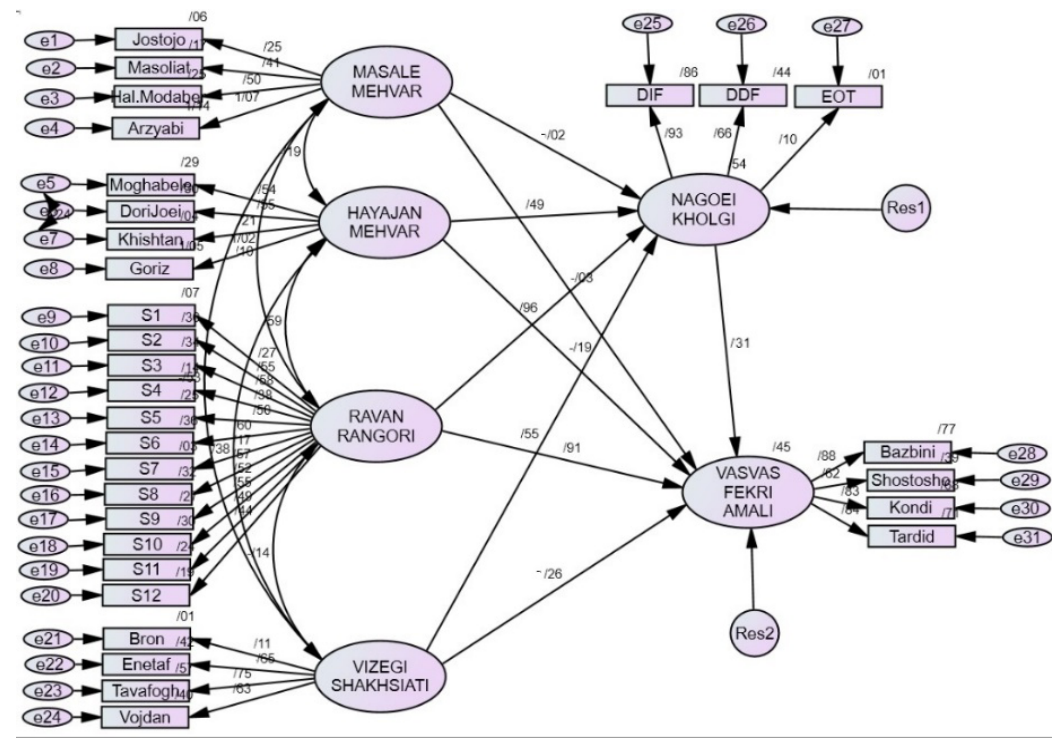

أنتوانث

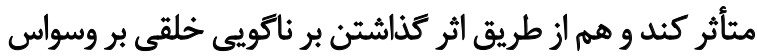

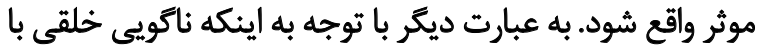

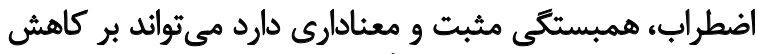

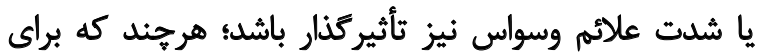

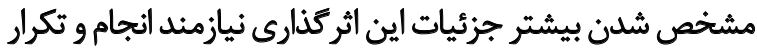

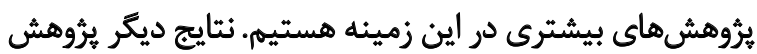

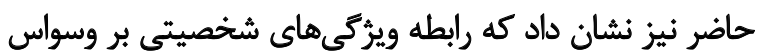

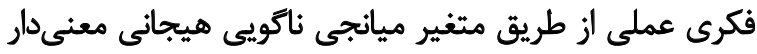

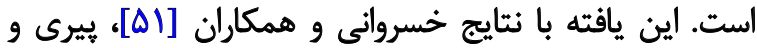

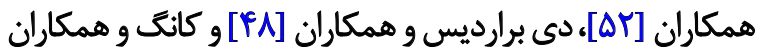

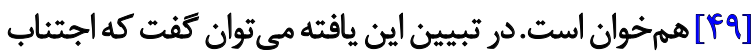

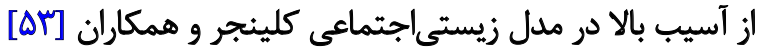

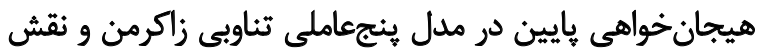

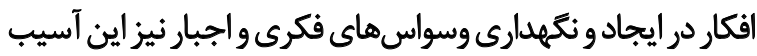

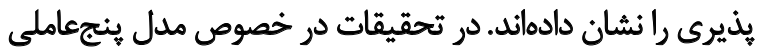

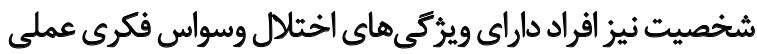

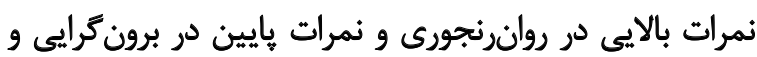

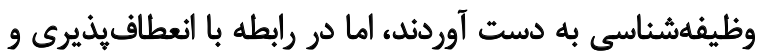

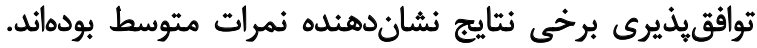

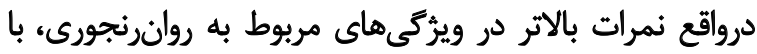

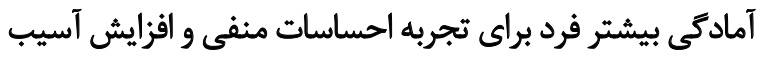

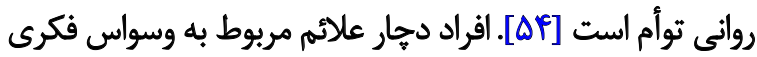

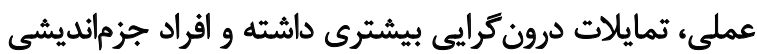

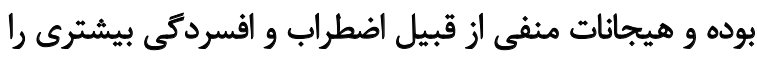

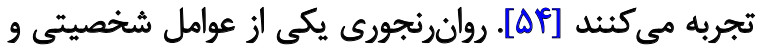

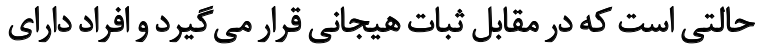

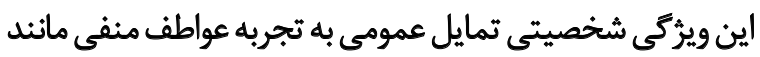

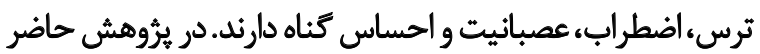

تصوير ا. مدل ساختارى فرضيات يرؤهش بانمايش ضرايب مسير

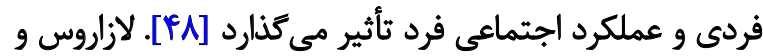

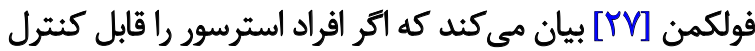

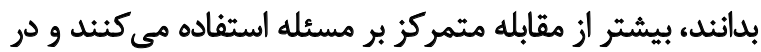

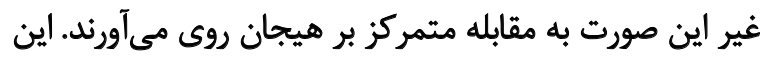

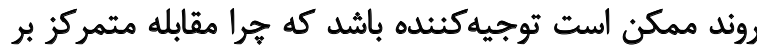

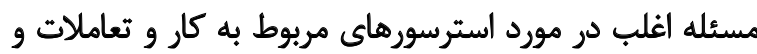

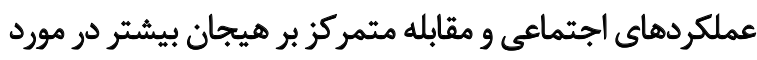

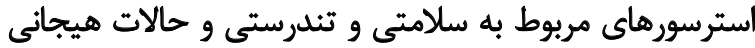

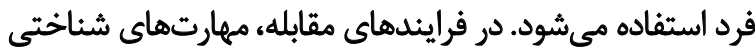

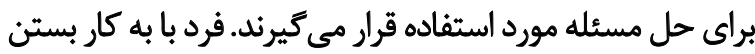

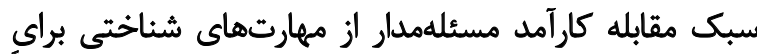

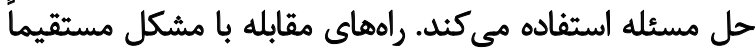

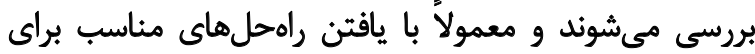

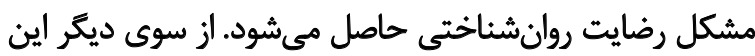

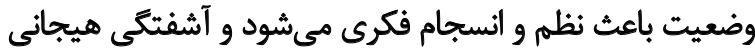

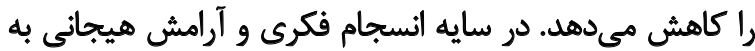

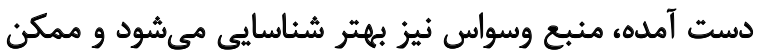

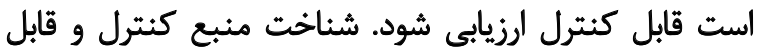

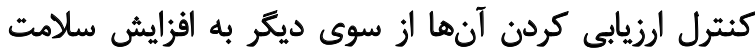

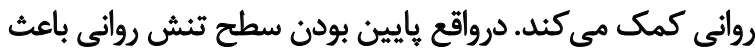

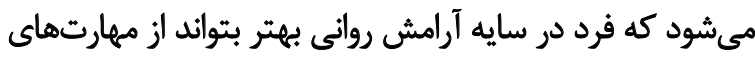

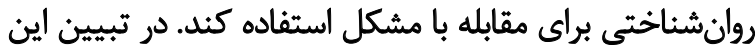

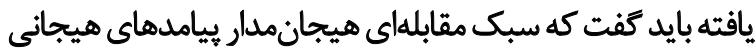

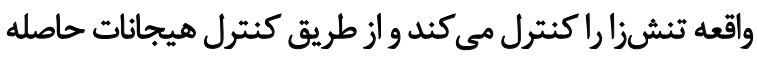

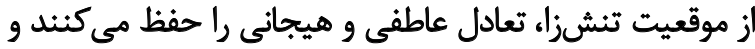

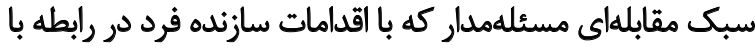

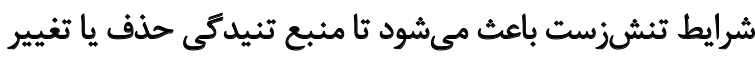

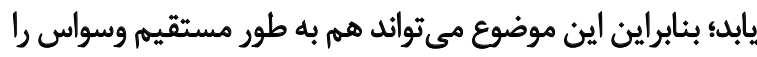


شخصيتى واختلالات روانى همراه با اين بيمارى توجه كنند؛ زيرا

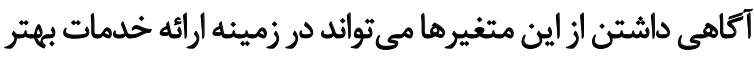
و مناسبتر به اين بيماران كمك إنينده باشيد.

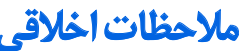

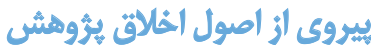

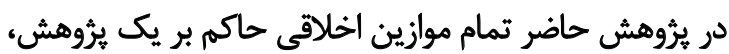

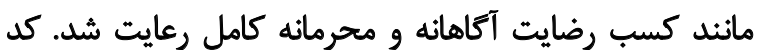
اخلاق IR.KMU.REC.1398.527 است.

$$
\text { Jlo }
$$

الين مقاله حاصل ياياننامه دكترى روانشناسى عمومى خانم مريم يور امر المهى در دانشكاه باهنر، دانشكده ادبيات و علوم انساني عماني

\section{مشاركت ثويسند انتان}

تمامى نويسندكان در نكارش اثر به يك اندازه مشاركت

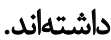

\section{ت تعارض مثاقع}

بنابر اظهار نويسندكًان اين مقاله هيج تضاد منافعى ندارد.

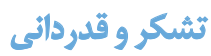

از معاونت محترم يثروهشى دانشكاه آزاد اسلامى زرند و دانشكاه علوميزشكى كرمان تقدير مى مشود.
نيز بين برون كرايى و وسواس ارتباطى يافت نشد. به بيان ديكًر

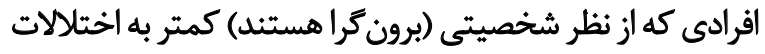

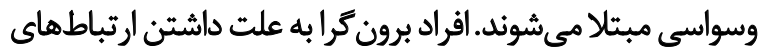

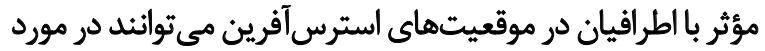

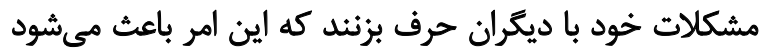

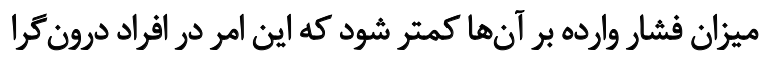

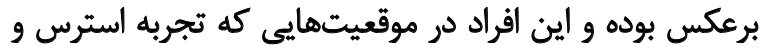
ناراحتى دارند در سطح شناختى از موضوع، فاجعهسازي مى كئند

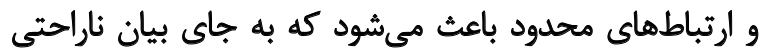

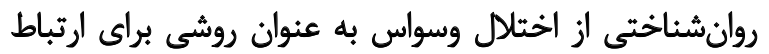

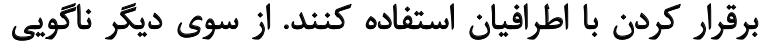

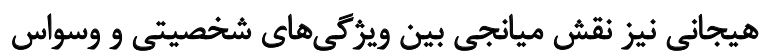

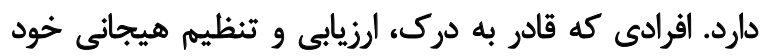

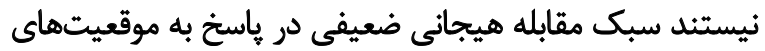

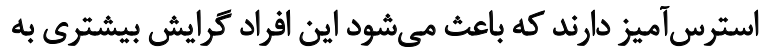

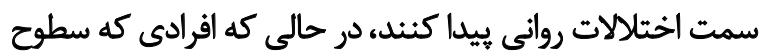

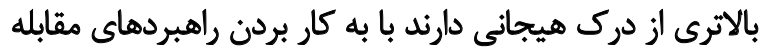

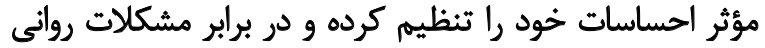

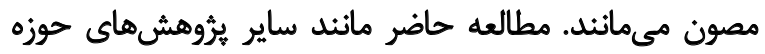

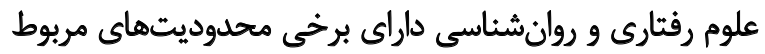
به صحت و تعميميذيرى نتايج است. تثيجليرى در نهايت مىتوان نتيجه كرفت كه مدليابى سبكهاى

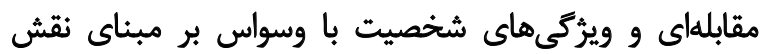

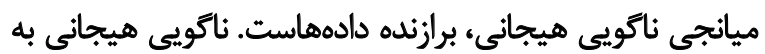

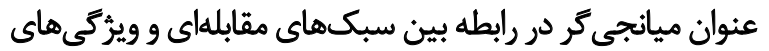

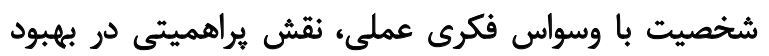
سلامث روان بيماران وسواسى دارد.

از محدوديتهاي يرؤهش حاضر اين بود كه از ابزارهاي

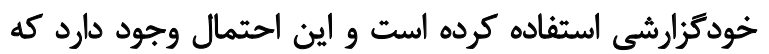

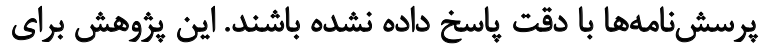

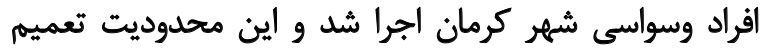

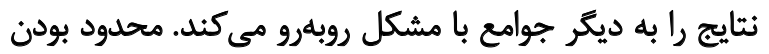

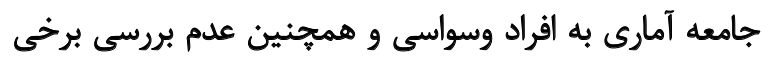

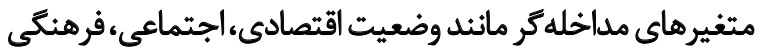

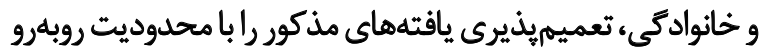

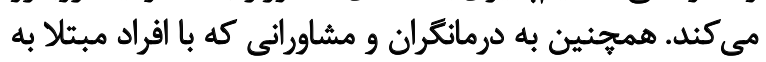

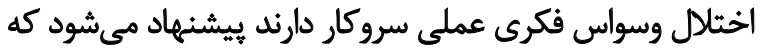

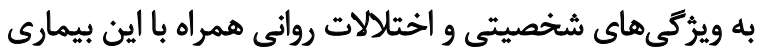

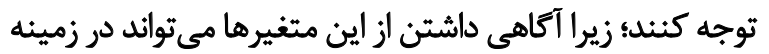

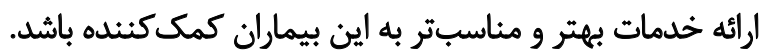

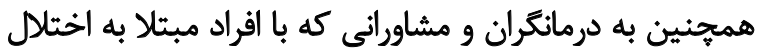

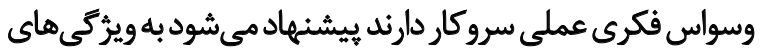




\section{References}

[1] Naghdi S, Bakhshipour A, Bafandeh H. [A comparative study of personality trait in obsessive -compulsive disorder and normal individuals (Persian)]. Journal of Instruction and Evaluation. 2013; 6(23):91-104. http:// jinev.iaut.ac.ir/article_521494.html

[2] Abramowitz JS, Deacon BJ, Olatunji BO, Wheaton MG, Berman NC, Losardo $D$, et al. Assessment of obsessive-compulsive symptom dimensions: Development and evaluation of the Dimensional Obsessive-Compulsive Scale. Psychological Assessment. 2010; 22(1):180-98. [DOI:10.1037/a0018260] [PMID]

[3] De Houwer J, Teige-Mocigmba S, Spruyt A, Moors A. Implict measures: A normative analysis and review. Psychological Bulletin. 2009; 135(3):347-68. [DOI:10.1037/a0014211] [PMID]

[4] Almardani Some'eh S. [The comparison of Marital Satisfaction in OCDpateints and normal individuals (Persian)]. Shenakht Journal of Psychology and Psychiatry. 2019; 5(6):13-23. [DOI:10.29252/shenakht.5.6.13]

[5] American Psychiatric Association. Diagnostic and statistical manual of mental disorders: DSM-IV [Internet]. 1994 [Updated 2010 March 8]. Available from: https://www.nlm.nih.gov/research/umls/sourcereleasedocs/current/DSM4/index.html

[6] Hasanzadeh A. [Comparison of metacognitive components in adolescents with and without obsessive-compulsive disorder (Persian)]. Tehran: Allameh Tabataba'i University; 2011. https://ganj.irandoc.ac.ir//\#/ articles/dd6a8b5351ba208506879f57e9173aca

[7] Sohrabzadeh Fard A, Hakim Shooshtari M, Alibaba S. [Comparison of psychopathology and personality traits in three groups of mothers of children with anxiety disorders, obsessive-compulsive disorder and healthy group (Persian)]. Iranian Journal of Psychiatry and Clinical Psychology. 2018; 24(2):164-75. [DOI:10.32598/ijpcp.24.2.164]

[8] Sajadmanesh R. [The relationship between personality traits and perceived stress with coping stress styles in Ahwaz Payame Noor University Staff (Persian)]. Ahvaz: Azad Eslami University; 2012.

[9] Hamidi F, Shirzad Aski M. [Relationship between personality characteristics and metacognitive strategies with computer self-efficacy in student-teachers (Persian)]. Information and Communication Technology in Educational Sciences. 2016; 6(4):23-38. http://ictedu.iausari.ac.ir/ article_652262.htm

[10] Bakhshayesh AR. [The relationship between personality types and general health with job satisfaction of Yazd health center staffs (Persian)]. Payavard Salamat. 2013; 7(1):42-55. http://payavard.tums.ac.ir/ article-1-5017-en.htm

[11] Dixon-Gordon KL, Conkey LC, Whalen DJ. Recent advances in understanding physical health problems in personality disorders. Current Opinion in Psychology. 2018; 21:1-5. [DOI:10.1016/j.copsyc.2017.08.036] [PMID] [PMCID]

[12] Razagh Pour M, Hoseinzadeh AA. [The mediating role of Cognitive Emotion Regulation Strategies in the relationship between personality traits with somatization symptoms of female university students (Persian)]. Quarterly of Applied Psychology. 2019; 13(2):295-316. http:// apsy.sbu.ac.ir/article_97219.html

[13] McCrae RR, Costa Jr PT. A contemplated revision of NEO five-factor inventory. Personality and Individual Differences. 2004; 36(3):587-96. [DOI:10.1016/S0191-8869(03)00118-1]

[14] Dehghanzadeh E, Esmailishad B. [Prediction of student creativity upon personality traits and learning strategies (Persian)]. Educational Development of Judishapur. 2019; 10 Suppl:102-13. http://journals.ajums. ac.ir/article_92597.html
[15] Rector NA, Hood K, Richter MA, Michael Bagby R. Obsessive-compulsive disorder and the five-factor model of personality: Distinction and overlap with major depressive disorder. Behaviour Research and Therapy. 2002; 40(10):1205-19. [DOI:10.1016/S0005-7967(02)00024-4]

[16] Ghazanfari F, Kadampoor A. [The relationship between mental health and coping strategies in citizenship of Khoramabad city (Persian)]. Journal of Fundamentals of Mental Health. 2008; 10(37):47-54. [DOI:10.22038/JFMH.2008.1775]

[17] Khaledian H, Parhizkar B, Shikhesmaeili F, Moradi M. [Comparison of the personality characteristics and coping strategies between the patients with irritable bowel syndrome and normal individuals (Persian)] Scientific Journal of Kurdistan University of Medical Sciences. 2019; 24(3):85-95. [DOI:10.29252/sjku.24.3.85]

[18] Volkmar FR, Paul R, Cohen D. Handbook of autism and pervasive developmental disorders. Hoboken: John Wiley \& Sons; 2006. https:// books.google.com/books?id=r6z7CT5v8L4C\&printsec=frontcover\&dq=

[19] Higgins DJ, BaileySR, Pearce JC. Factors associated with functioning style and coping strategies of families with a child with an autism spectrum disorder. Autism. 2005; 9(2):125-37. [DOI:10.1177/1362361305051403] [PMID]

[20] Hooshyar M, Kakavand A, Ahmadi A. [The effectiveness of mindfulness-based therapy on quality of life and coping strategies of mothers of children with autistic spectrum disorder (Persian)]. Quarterly Journal of Social Work. 2018; 7(2):32-9. http://socialworkmag.ir/article-1-392-en. $\mathrm{html}$

[21] Carver CS, Pozo C, Harris SD, Noriega V, Scheier MF, Robinson DS, et al. How coping mediates the effect of optimism and distress: A study of women with early stage breast cancer. Journal of Personality and Social Psychology. 1993; 65(2):375-90. [DOI:10.1037/0022-3514.65.2.375] [PMID]

[22] Ghassem Boroujerdi F, Sohrabi F, Borjali A. [Evaluation of the interactive relationship between attachment styles, coping styles and mental health in adolescents (Persian)]. Journal of Social Psychology (New Findings in Psychology). 2012; 7(21):85-100. https://www.sid.ir/fa/journal/ ViewPaper.aspx?ID=160177

[23] Folkman S. Stress: Appraisal and coping. In: Gellman MD, Turner JR, editors. Encyclopedia of Behavioral Medicine. New York, NY: Springer 2013. [DOI:10.1007/978-1-4419-1005-9_215]

[24] Rahimi B. [The relationship between Meaning of Life and coping strategies with happiness in students with depressed and normal people of Kurdistan University [MA. thesis]. Urmia: Urmia University; 2015.

[25] Sadock VA, Sadock S. Comprehensive textbook of psychiatry. Philadelphia: Lippincott Williams \& Wilkins; 2015.

[26] Sanderson C. Health psychology [F Jomhouri, F Meschi, Sh Sodager, Persian trans.]. Karaj: Sarafraz Publications; 2015.

[27] Lazarus RS, Folkaman S. Stress, appraisal and coping. New York, NY: Springer; 1984. https://books.google.com/books?id=iySQQuUpr8C\&dq

[28] Ramandi M, Shareh H, Siltani E, Khormaee F, Mirzaee A. [Role of coping strategies in the psychological problems of patients with diabetes (Persian)]. Scientific Journal of llam University of Medical Sciences. 2013; 21(3):163-73. http://sjimu.medilam.ac.ir/article-1-1208-en.html

[29] Besharat MA, Barati N, Lotfi J. [Relationship between coping styles and mental health in a sample of multiple sclerosis patients (Persian)]. Research in Medicine. 2008; 32(1):27-35. http://pejouhesh.sbmu.ac.ir/ article-1-457-en.html 
[30] Wingenfeld K, Mensebach C, Rullkoetter N, Schlosser N, Schaffrath $\mathrm{C}$, Beblo T, et al. Relationship between coping with negative life-events and psychopathology: Major depression and borderline personality disorder. Psychology and Psychotherapy: Theory, Research and Practice. 2009; 82(4):421-5. [DOI:10.1348/147608309X452416] [PMID]

[31] Nargesi F, Fathi-Ashtiani A, Davoudi I, Ashrafi E. [The mediating role of difficulties in emotion regulation strategies on the relationship between alexithymia, anxiety sensitivity and distress tolerance with obsessivecompulsive symptoms (Persian)]. Middle Eastern Journal of Disability Studies. 2018; 8:9. http://jdisabilstud.org/article-1-1103-en.html

[32] Heshmati R, Ghorbani N, Rostami R, Ahmadi M, Akhavan H. [Comparative study of alexithymia in patients with psychotic disorders, non psychotic and normal people (Persian)]. Avicenna Journal of Clinical Medicine. 2010; 17(1):56-61. http://sjh.umsha.ac.ir/article-1-290-en.html

[33] Luminet O, Vermeulen N, Demaret C, Taylor GJ, Michael Bagby R. Alexithymia and level of processing: Evidence for an overall deficit in remembering emotion words. Journal of Research in Personality. 2006; 40(5):713-33. [DOI:10.1016/j.jrp.2005.09.001]

[34] Scarpazza C, di Pellegrino G, Làdavas E. Emotional modulation of touch in alexithymia. Emotion. 2014; 14(3):602-10. [DOI:10.1037/a0035888] [PMID]

[35] van der Velde J, Gromann PM, Swart M, Wiersma D, de Haan L, Bruggeman $\mathrm{R}$, et al. Alexithymia influences brain activation during emotion perception but not regulation. Social Cognitive and Affective Neuroscience. 2015; 10(2):285-93. [DOI:10.1093/scan/nsu056] [PMID] [PMCID]

[36] Taylor GJ, Parker JD, Bagby RM. Relationships between alexithymia and related constructs. In: Vingerhoets A, Van Bussel F, Boelhouwer J, editor. The (non)- expression of emotions in health and disease. Tilburg: Tilburg University Press; 1999.

[37] Mattila AK, Luutonen S, Ylinen M, Salokangas RKR, Joukamaa M. Alexithymia, human relationships, and mobile phone use. The Journal of Nervous and Mental Disease. 2010; 198(10):722-7. [DOI:10.1097/ NMD.0b013e3181f4ab50] [PMID]

[38] Besharat MA. Reliability and factorial validity of a Farsi version of the 20-item Toronto Alexithymia Scale with a sample of Iranian students. Psychological Reports. 2007; 101(1):209-20. [DOI:10.2466/ pr0.101.1.209-220] [PMID]

[39] Grabe HJ, Ruhrmann S, Ettelt S, Müller A, Buhtz F, Hochrein A, et al. Alexithymia in obsessive-compulsive disorder - results from a family study. Psychotherapy and Psychosomatics. 2006; 75(5):312-8. [DOI:10.1159/000093954] [PMID]

[40] Hooman HA. [Structural equation modeling using laser software (Persian)]. Tehran: Samat Publications. 2005. https://www.adinehbook. com/gp/product/9644599624

[41] Folkman S, Lazarus RS, Gruen RJ, DeLongis A. Appraisal, coping, health status, and psychological symptoms. Journal of Personality and Social Psychology. 1986; 50(3):571-9. [DOI:10.1037/0022-3514.50.3.571]

[42] Agha Yousefi AR. [The role of personality factors on coping strategies and the impact of treatment methods on personality factors and depression (Persian)] [PhD. dissertation]. Tehran: Tarbiat Modares University; 2001. https://ganj.irandoc.ac.ir//\#/articles/fcdaca9addd68368df18de45509bfb04

[43] Kiamehr J. [Standardization of the short form of the NEO five factor questionnaire and its factor structure among students of Tehran university (Persian)]. Tehran: Allameh Tabataba'i University; 2003. http://d-lib. atu.ac.ir/site/catalogue/66810
[44] Michael Bagby R, Parker JDA, Taylor GJ. The twenty-item Toronto Alexithymia scale: I. Item selection and cross-validation of the factor structure. Journal of Psychosomatic Research. 1994; 38(1):23-32. [DOI:10.1016/0022-3999(94)90005-1]

[45] Besharat MA, Masoodi M, Gholamali Lavasani M. [Alexithymia and psychological and physical vulnerability (Persian)]. Thoughts and Behavior in Clinical Psychology. 2015; 9(34):47-56. https://jtbcp.riau.ac.ir/ article_107.html

[46] Rachman SJ, Hodgson RJ. Obsessions and compulsions. New Jersey: Prentice Hall; 1997.

[47] Habibi Kaleybar R, Mohammadzadegan R, Afi E. [Comparison of alexithymia and impulsive behaviors in schizophrenia patients and healthy individuals (Persian)]. Quarterly of the Horizon of Medical Sciences. 2017; 23(3):199-205. http://hms.gmu.ac.ir/article-1-2634-en.html

[48] De Berardis D, Serroni N, Campanella D, Rapini G, Olivieri L, Feliziani $B$, et al. Alexithymia, responsibility attitudes and suicide ideation among outpatients with obsessive-compulsive disorder: An exploratory study. Comprehensive Psychiatry. 2015; 58:82-7. [DOI:10.1016/j. comppsych.2014.12.016] [PMID]

[49] Kang JI, Namkoong K, Yoo SW, Jhung K, Kim SJ. Abnormalities of emotional awareness and perception in patients with obsessive - compulsive disorder. Journal of Affective Disorders. 2012; 141(2-3):286-93. [DOI:10.1016/j.jad.2012.04.001] [PMID]

[50] Davis SK, Humphrey N. The influence of Emotional Intelligence (EI) on coping and mental health in adolescence: Divergent roles for trait and ability El. Journal of Adolescence. 2012; 35(5):1369-79. [DOI:10.1016/j. adolescence.2012.05.007] [PMID]

[51] Khosravani V, Samimi Ardestani M, Sharifi Bastan F, Kamali Z. The relationship between alexithymia and symptom dimensions in patients with obsessive-compulsive disorder. Journal of Obsessive-Compulsive and Related Disorders. 2017; 14:127-33. [DOI:10.1016/j.jocrd.2017.04.001]

[52] Piri Z, Amiri Majd M, Bazzazian S, Ghamari M. [The mediating role of coping strategies in the relationship of difficulties in emotion regulation with internet addiction among college students (Persian)]. Quarterly of the Horizon of Medical Sciences. 2019; 26(1):38-53. http://hms.gmu. ac.ir/article-1-3262-en.html

[53] Robert Cloninger C. A systematic method for clinical description and classification of personality variants. A proposal. Archives of General Psychiatry. 1987; 44(6):573-88. [DOI:10.1001/ archpsyc.1987.01800180093014] [PMID]

[54] Paunonen SV, Ashton MC. Big five factors and facets and the prediction of behavior. Journal of Personality and Social Psychology. 2001; 81(3):524-39. [DOI:10.1037/0022-3514.81.3.524] [PMID] 